\title{
Appetitive odor learning does not change olfactory coding in a subpopulation of honeybee antennal lobe neurons
}

\author{
P. Peele $\cdot$ M. Ditzen $\cdot$ R. Menzel $\cdot$ C. G. Galizia
}

\begin{abstract}
Odors elicit spatio-temporal patterns of activity in the olfactory bulb of vertebrates and the antennal lobe of insects. There have been several reports of changes in these patterns following olfactory learning. These studies pose a conundrum: how can an animal learn to efficiently respond to a particular odor with an adequate response, if its primary representation already changes during this process? In this study, we offer a possible solution for this problem. We measured odor-evoked calcium responses in a subpopulation of uniglomerular AL output neurons in honeybees. We show that their responses to odors are remarkably resistant to plasticity following a variety of appetitive olfactory learning paradigms. There was no significant difference in the changes of odor-evoked activity between single and multiple trial forward or backward conditioning, differential conditioning, or unrewarded successive odor stimulation. In a behavioral learning experiment we show that these neurons are necessary for conditioned odor responses. We conclude that these uniglomerular projection neurons are necessary for reliable odor coding and are not
\end{abstract}

P. Peele - M. Ditzen - R. MenzelC. G. Galizia Institute of Neurobiology, Freie Universität Berlin, 14195 Berlin, Germany

C. G. Galizia

Department of Entomology, University of California, Riverside, CA 92521, USA

Present Address:

C. G. Galizia (凶)

Universität Konstanz, 78457 Konstanz, Germany

e-mail: Giovanni.Galizia@uni-konstanz.de modified by learning in this paradigm. The role that other projection neurons play in olfactory learning remains to be investigated.

Keywords Learning and memory - Olfactory coding . Calcium imaging $\cdot$ Projection neurons · Coding invariance
Abbreviations
$\mathrm{AL}$
Antennal lobe
CR
Conditioned response
$\mathrm{CS}-$
Non-rewarded stimulus
$\mathrm{CS}+$
Rewarded (conditioned) stimulus
ITI
Inter-trial interval
1-ACT
Lateral antenno-cerebralis tract
LP
Lateral protocerebrum
m-ACT Medial antenno-cerebralis tract
MB Mushroom body
ml-ACT Mediolateral antenno-cerebralis tract
OB Olfactory bulb
OSN Olfactory sensory neuron
PN Projection neuron
RM ANOVA Repeated measures analysis of variance
SEG Subesophageal ganglion
T1-T4 Tracts $1-4$ in the antennal lobe
US Unconditioned stimulus
PER Proboscis extension reflex
VUMmx1 Ventral unpaired neuron \# 1 of the maxillary neuromere

\section{Introduction}

The internal representation of the external world is an inherent and fundamental function of all nervous 
systems that needs to be reliable to ensure the generation of adequate behavioral responses. This is also true for the chemical senses, which play a vital role for most animals. Primary olfactory information is processed in the vertebrate olfactory bulb (OB) and its insect analogue, the antennal lobe (AL) (Hildebrand and Shepherd 1997). Both are subdivided into functional processing units, the olfactory glomeruli. Odors elicit specific spatio-temporal combinatorial patterns of activated glomeruli in vertebrate $\mathrm{OBs}$ and invertebrate ALs (Galizia and Menzel 2001; Friedrich 2002). A particular glomerulus receives afferent input by a specific olfactory sensory neuron type (OSN). Local neurons within the $\mathrm{OB}$ or the AL modify the incoming activity (Malun 1991; Yokoi et al. 1995; Mori et al. 1999; Sachse and Galizia 2002), and the processed information is relayed to higher order brain centers by vertebrate mitral/tufted cells or the insect projection neurons (PNs). Higher order olfactory neuropiles in insects are the mushroom bodies (MB) and the lateral protocerebrum (LP) (Mobbs 1982; Abel et al. 2001; Müller et al. 2002; Marin et al. 2002; Wong et al. 2002; Tanaka et al. 2004). In the honeybee, OSN afferents travel in four different tracts, $\mathrm{T} 1-\mathrm{T} 4$, that innervate about 160 glomeruli altogether. PNs run in three different antenno-cerebral tracts $(\mathrm{ACT})$. The medio-lateral ACT (ml-ACT) contains pluriglomerular cells. The lateral and median ACTs (1-and m-ACTs) contain axons of uniglomerular PNs (Abel et al. 2001). It should be noted that the nomenclature for PN tracts differs for different insect species. For example, the location of the honeybee 1-ACT corresponds to the Drosophila oACT, and whether the two are homologous remains to be investigated.

Vertebrates as well as many invertebrates can be conditioned to olfactory stimuli (Menzel 1990; Wilson and Stevenson 2003; Davis 2004). Sucrose stimulation of antennal or proboscis chemoreceptors of a hungry bee leads to the proboscis extension response (PER). In an appetitive olfactory learning paradigm (PER conditioning), an odor, the conditioned stimulus (CS), is paired with a subsequent sucrose reward as the unconditioned stimulus (US). The animals form an association between the two, so that an odor stimulation alone elicits the PER (conditioned response, CR) previously elicited only by the US. This effect is clearly associative and involves classical conditioning (Bitterman et al. 1983). The number of conditioning trials applied to the honeybee influences which kind of memory is induced. A single conditioning trial results in short-term memory which decays rapidly and is sensitive to amnesic treatments. This memory is independent of translation and transcription. In contrast, multiple conditioning trials induce a stable, long-term memory that needs both translation and transcription (Menzel 1999).

The cellular changes leading to learning appear to be dispersed among different areas of the brain (Menzel 1990; Gluck and Granger 1993; Tully et al. 1994; Hammer and Menzel 1998; Menzel 1999). Amongst others, neural plasticity due to olfactory learning has been found at the level of the first processing station in the olfactory pathway, in the vertebrate OB (Woo et al. 1987; Sullivan and Wilson 1995; Johnson et al. 1995; Brennan et al. 1998) and the insect AL (Faber et al. 1999; Sandoz et al. 2003; Yu et al. 2004; Daly et al. 2004). Changes at the first odor processing level are likely to affect the neural representation of an odor. But how can an animal learn to respond to a particular odor if its neural representation is changing? Moreover, behavioral phenomena such as contextual, structural and configural learning require a stable representation of stimulus features (for example, that of an odor) in different circuits that are selectively and specifically combined (Gerber and Menzel 2000; Sandoz and Menzel 2001; Menzel and Giurfa 2001; Deisig et al. 2001). We therefore investigated the effects of associative learning on the representation of olfactory information in the AL. To this end, we combined a variety of appetitive olfactory conditioning paradigms with in vivo calcium imaging of one subpopulation of the olfactory PNs, the 1ACT PNs (Sachse and Galizia 2002). We measured odor responses before, during and after associative olfactory learning, and found that odor representation in these cells is reliable and stable throughout appetitive learning experiments.

\section{Methods}

\section{Preparation}

Adult foraging bees, Apis mellifera carnica, were caught at the hive in the afternoon 1 day before the experiments. Bees were experimentally naïve, but may have experienced odors during foraging flights. They were cooled for anesthesia, fixed in a recording stage (Galizia et al. 1997), and fed with sucrose water (30\%) until satiation. Bees were kept at $20^{\circ} \mathrm{C}$ in a dark humidified box over night. The next day PNs were stained as follows: a window was cut in the head capsule and glands and trachea were carefully removed to allow visual access to the brain. A glass electrode was coated with crystals of FURA-dextran (potassium salt, $10.000 \mathrm{MW}$, Invitrogen, Germany), dissolved in $3 \%$ bovine serum albumin solution (Albumin Fraction V, 
AppliChem GmbH, Germany), and injected into the right deutocerebrum dorsolateral to the alpha-lobe, aiming for the I-ACT. Using different activity indicators including calcium dyes, other studies have successfully demonstrated plasticity in neural signaling in vertebrates and invertebrates (Faber et al. 1999; Faber and Menzel 2001; Yu et al. 2004; Mutoh et al. 2005; Lohmann and Wong 2005). This indicates that calcium sensitive dyes do not interfere with the intracellular signaling cascades to a degree that would prevent any modulatory effects. The dye was left to travel along the tract for $4-8 \mathrm{~h}$. Then, bees were prepared by fixing the antennae with soft dental wax (Kerr, Sybron Dental Specialties, USA). Animals were immobilized by fixing the thorax and abdomen to the recording chamber with wax (Deiberit 502, Dr Boehme\&Schoeps, Germany). The brain was rinsed with Ringer solution $(130 \mathrm{mM}$ $\mathrm{NaCl}, 6 \mathrm{mM} \mathrm{KCl}, 4 \mathrm{mM} \mathrm{MgCl} 2,5 \mathrm{mM} \mathrm{CaCl}_{2}, 160 \mathrm{mM}$ sucrose, $25 \mathrm{mM}$ glucose, $10 \mathrm{mM}$ HEPES, pH 6.7, 500 mOsmol; all chemicals from Sigma-Aldrich, Germany). Strong movements of the brain needed to be prevented to achieve stable imaging experiments. Therefore, a second hole was cut ventrally to the antennae and the compact structure of muscles, esophagus, and supporting chitin was lifted and put under slight tension (Mauelshagen 1993). Thus stabilization was accomplished without damage to the brain. However, this preparation impairs the PER. To allow for sucrose stimulation the proboscis was manually extended and placed on a small glass capillary that was fixed to the recording chamber. After surgery and before recording the bees were left to recover for at least $30 \mathrm{~min}$.

\section{Imaging}

Imaging was done using a CCD based imaging system (Polychrome IV with Imago-QE camera, T.I.L.L. Photonics, Germany). Monochromatic excitation light alternated between 340 and $380 \mathrm{~nm}$. Fluorescence was detected at a sampling rate of $5 \mathrm{~Hz}$ with a fluorescence microscope (Olympus BX-50WI, Japan) equipped with a 20×, NA $=0.95$ dip objective (Olympus), 505 DRLPXR dichroic mirror and $515 \mathrm{~nm}$ LP filter (Omega Filters, VT, USA). Resolution was $172 \times 130$ pixels binned on chip from $1,376 \times 1,040$ pixels, resulting in a spatial sampling rate of $2.4 \mu \mathrm{m}$ per pixel side.

Odors were diluted in mineral oil to $1 \%$ (1-hexanol) or $0.1 \%$ (1-octanol and 1-nonanol, all odors from Sigma-Aldrich, Germany). Five microliter of the odor solution were applied onto a $1 \mathrm{~cm}^{2}$ piece of filter paper and placed in a plastic syringe. Odors were injected into a continuous air stream directed to the antennae using a computer controlled olfactometer (Galizia et al. 1997).

For the US we delivered a drop of $2 \mu \mathrm{l} 30 \%$ aqueous sucrose solution to the antennae and the proboscis, using a $10 \mu 1$ pipette with standard tips.

\section{Data analysis}

All data were analyzed using custom software written in IDL (Research systems, CO, USA). First, the raw fluorescent images were manually corrected for movement within each measurement and for shifts between measurements. Scattered light correction was applied to avoid that glomeruli with strong activity would cause fictive activity in neighboring silent glomeruli (Galizia and Vetter 2004). We calculated $F_{i}^{\prime}=F_{i}+n\left[F_{i}-\operatorname{sm}\left(F_{i}\right)\right]$, where $F_{i}$ is image number $i$, and $\operatorname{sm}\left(F_{i}\right)$ the same image after application of a spatial low-pass filter. We used a boxcar average filter with kernel size of $20 \mu \mathrm{m}$, i.e. less than the radius of one glomerulus. The images $F_{i}^{\prime}$ are the scattered-light corrected images. The number $n$ gives the strength of the applied filter. In this study, we found $n=3$ to give the best results, as compared to $n=1$ in previous work (Galizia and Vetter 2004). Note that this procedure does not change the measured values in areas where there is no spatial contrast: in these areas, the lowpassed filtered image has the same value as the original image, and consequently $F_{i}^{\prime}=F_{i}$.

For each image $i$, we then calculated the percentage ratio $R_{i}=\left(F_{\mathrm{i}} 340 \mathrm{~nm} / F_{i} 380 \mathrm{~nm}\right) \times 100$. Background ratio was determined by an average of five frames obtained before stimulation and was subtracted from every ratio frame of a measurement, thereby setting ratio values to 0 just before stimulus onset. The resulting values are proportional to intracellular calcium concentration changes, but are measured in arbitrary units, since we could not calibrate absolute calcium concentration.

The morphological structure allowed us to identify glomeruli on the basis of their borderlines using the digital atlas of the AL as a reference (Galizia et al. 1999a). This method has already been described in detail (Sachse et al. 1999; Galizia et al. 1999b). Honeybee glomeruli are labeled with a number and the name of the antennal nerve tract that innervates them, ranging from T1 to T4, e.g. T1-28 or T3-45 (Flanagan and Mercer 1989). Since all glomeruli in this study were from the $T 1$ tract, we have simplified their names by omitting $\mathrm{T} 1$ from the name. We analyzed responses in glomeruli 17 , $23,25,28,29,33,35,36,37,38,42,47,48,49$ and 60 . "Glomerular activity" in this study specifically refers to activity of the uniglomerular PNs in each glomerulus, because only these neurons were stained, and therefore 
only this particular population of cells was measured in each glomerulus. For each identified glomerulus time courses were averaged taking an area of $9 \times 9$ pixels (corresponding to $21.6 \mu \mathrm{m} \times 21.6 \mu \mathrm{m}$ always within the glomerulus chosen). Glomerular calcium response magnitude (hereafter simply "response") was calculated as the mean of the time course during odor stimulation. Statistical analysis was performed using Sigmastat (SPSS Inc, IL, USA).

For false-color display, responses were calculated for each pixel. The resulting image was median filtered in space $(3 \times 3$ pixels $)$.

\section{Plasticity of odor responses}

All experimental protocols are summarized in Fig. 1. 1nonanol was always the reinforced odor (CS). In one part of the experiments (set 1) we investigated changes of the responses to 1-nonanol due to repeated stimulations, and due to pairing of this odor with a sucrose reward in an absolute conditioning paradigm, with special focus on progression in time after the conditioning phase. Thereafter, we included 1-hexanol and 1octanol into the stimulation protocols, allowing us to examine the effects of absolute conditioning on the representation of non-conditioned odors and the effect of differential conditioning on all odors involved (set 2).

\section{Single odor experiments}

We investigated the effects of absolute conditioning on the neural representation of the odor 1-nonanol (Fig. 1, set 1). The experiments consisted of three parts: odor responses before conditioning ("pre"), conditioning, and odor responses after conditioning ("post"). Odor stimulation was always $3 \mathrm{~s}$ long. We determined the "naïve" response to 1-nonanol in the "pre"-phase during which all animals received two presentations of 1-nonanol with an inter-trial interval (ITI) of 2 min. During conditioning a sucrose reward was applied for $3 \mathrm{~s}$ to the ipsilateral antenna of the imaged $\mathrm{AL}$ and to the proboscis. For forward pairing the odor preceded the reinforcement, for backward pairing that order was reversed, the overlap was $1 \mathrm{~s}$ in both cases. During the "post" phase all animals were presented with 1-nonanol after 1,5 and $15 \mathrm{~min}$ following conditioning.

Five groups were subject to the different conditioning protocols:

$1^{-} \quad 1$-trial unrewarded 1-nonanol $(n=9)$

$1^{\text {bw }} \quad 1$-trial backward conditioning $(n=14)$

$1^{+} \quad 1$-trial forward conditioning $(n=9)$ $3^{\text {bw }} 3$-trial backward conditioning with ITI of 2 min $(n=11)$

$3^{+} \quad 3$-trial forward conditioning with ITI of $2 \mathrm{~min}$ $(n=11)$.

This nomenclature indicates whether 1-nonanol was unrewarded $(-)$, forward paired with sucrose water $(+)$ or backward paired with sucrose water (bw). The number $(1,3)$ corresponds to how often the conditioning trials were repeated.

Calcium responses for each glomerulus were analyzed with a two-way repeated measures (RM) ANOVA, with the different conditioning groups as one factor and the "pre"-responses and "post"-responses at different points in time as the repeated factor. Multiple comparisons were performed using the Holms-Sidak post-hoc test.

\section{Multiple odor experiments}

We investigated the effect of sequential stimulation with three odors and differential conditioning (Fig. 1, set 2). Each experiment consisted of measuring odor responses before conditioning ("pre"), conditioning, and measuring odor responses after conditioning ("post"), similar to experiments of set 1. "Pre" and "post" consisted of three blocks with three odor stimulations each (1-hexanol, 1-octanol and 1-nonanol). Within "pre" and "post" blocks the odor sequence was pseudorandomized to prevent odor sequence effects (for example, an odor sequence could be $\mathrm{ABC}, \mathrm{CAB}, \mathrm{BCA}$, with each odor at a first, a second and a third position within the blocks). Different animals were tested with different sequences. We averaged the three "pre" responses and the three "post" responses to yield one "pre" and one "post" response to every odor which we then compared. CS-US pairing was done as for absolute conditioning (see above). Stimulus sequence within the conditioning block was identical for all animals within each group.

Seven groups were subject to the following conditioning protocols:

$3 \mathrm{~N}^{+} \quad 3$-trial absolute conditioning, ITI of $2 \mathrm{~min}$ (no CS-) $(n=10)$

$3 \mathrm{~N}^{+} \mathrm{O}^{-} \quad 3$-trial differential conditioning, ITI of $1 \mathrm{~min}$, CS+1-nonanol, CS-1-octanol $(n=9)$

$5 \mathrm{~N}^{+} \quad 5$-trial absolute conditioning, ITI of $2 \mathrm{~min}$ (no CS-) $(n=15)$

$5 \mathrm{~N}^{+} \mathrm{O}^{-} \quad 5$-trial differential conditioning, ITI of $1 \mathrm{~min}$, CS+1-nonanol, CS-1-octanol $(n=9)$

$5 \mathrm{~N}^{+} \mathrm{H}^{-} \quad$ 5-trial differential conditioning, $\mathrm{CS}+1$ nonanol, CS-1-hexanol $(n=10)$ 
Fig. 1 Stimulus protocols of the experiments. Stimulus duration for odors and sucrose was always $3 \mathrm{~s}$. During pairing, the two stimuli overlapped for $1 \mathrm{~s}$ Group labels reflect the respective conditioning protocol. The number gives how often the conditioning trial was repeated. $(-)$ stands for unrewarded odor, $(+)$ for forward pairing with sucrose and (bw) for backward pairing, 1-Nonanol was the only odor given throughout the first five groups (set 1) of the experiments. For the last seven groups (set 2), odors are indicated by capital letters: 1-hexanol $(H)$, 1-octanol $(O)$ and 1-nonanol $(N)$. Stimulations of the three odors were randomized in the "pre" and "post" phase

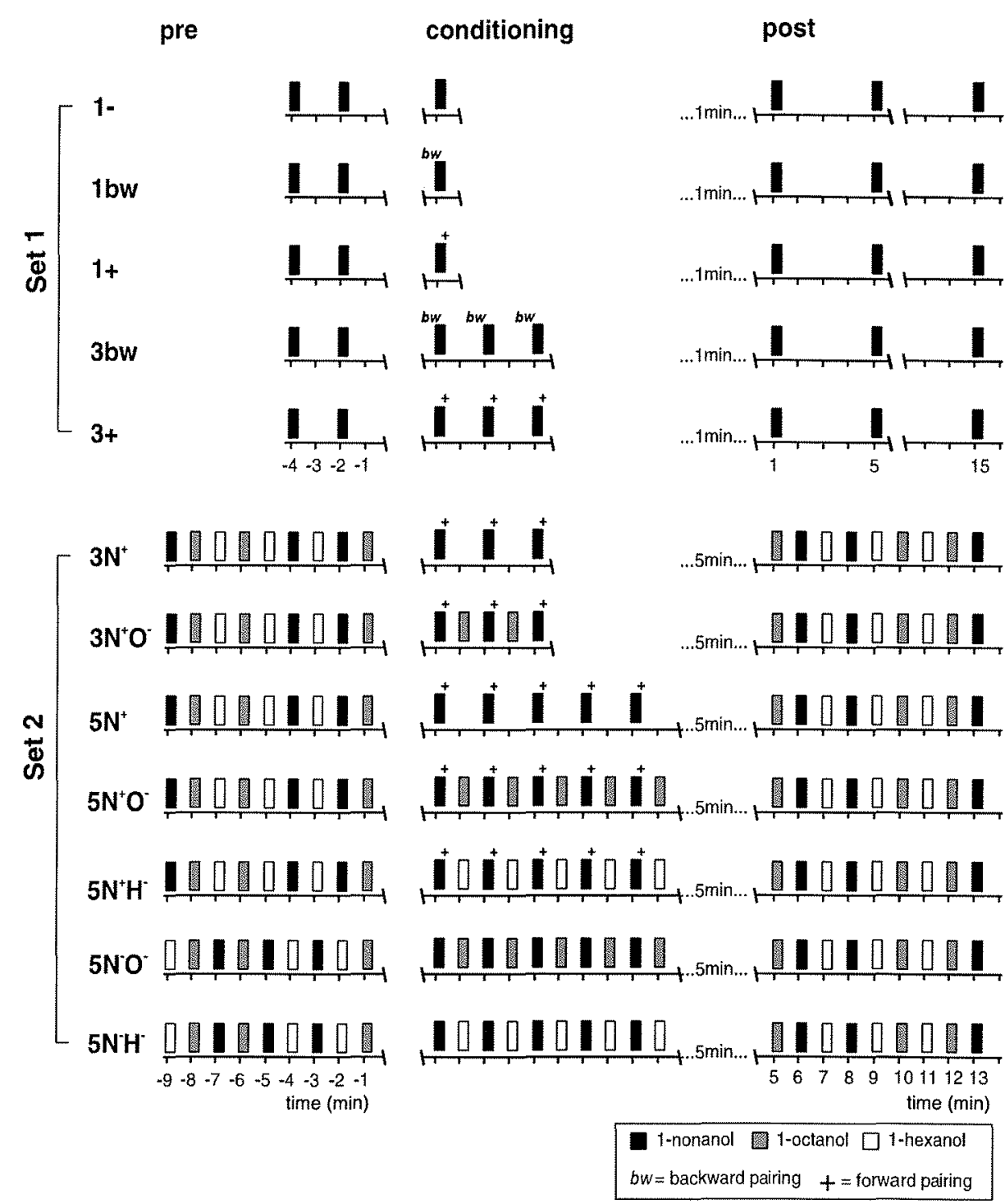

$5 \mathrm{~N}^{-} \mathrm{O}^{-} \quad 5$-trial stimulation as in $5 \mathrm{~N}^{+} \mathrm{O}^{-}$but without any reward $(n=10)$

$5 \mathrm{~N}^{-} \mathrm{H}^{-}$5-trial stimulation as in $5 \mathrm{~N}^{+} \mathrm{H}^{-}$but without any reward $(n=9)$.

In this nomenclature, the number $(3,5)$ indicates how often conditioning trials were repeated. Conditioned odors are indicated by capital letters: 1-hexanol $(\mathrm{H}), 1$ octanol $(\mathrm{O})$ and 1-nonanol $(\mathrm{N})$, with a $(+)$ for reinforced odor or a $(-)$ for unrewarded presentation. Thus, $5 \mathrm{~N}^{+} \mathrm{O}^{-}$represents a 5 -trial differential conditioning, with 1-nonanol reinforced and 1-octanol unrewarded.

Five minutes after conditioning the animals were subject to olfactory stimulation as described above ("post"), including all three odors 1-hexanol, 1-octanol and 1-nonanol.

Odor responses to the three odors in every glomerulus were analyzed with a two-way RM ANOVA, with the different conditioning groups as one factor and "pre"-responses and "post"-responses as the repeated factor time. Multiple comparisons were performed using the Holms-Sidak post-hoc test.

\section{Behavior}

The animals were prepared and stained as in the imaging experiments, with the difference that the esophagus and the muscles around the proboscis were not restrained in order to allow for the PER. First, 
animals were tested for PER to sucrose stimulation at each antenna, i.e. both the ipsilateral and the contralateral antenna to the stained 1-ACT PNs. Learning experiments started $30 \mathrm{~min}$ later to avoid any effects due to sensitization (Menzel et al. 1991).

Odor stimulation with 1-nonanol was again $3 \mathrm{~s}$, similar to the absolute conditioning experiments. Intertrial interval was 2 min throughout "pre" and conditioning. All animals received two "pre" odor stimulations. Thereafter the animals were conditioned by a 3-trial absolute conditioning as described above. Five minutes after conditioning the animals were tested for PER to 1nonanol stimulation at the ipsilateral antenna, at the contralateral antenna and at both antennae. The order of stimulation was randomized between animals. Stimulation of only one antenna was achieved by covering the other antenna with a sealed plastic tube. All animals were then examined for stained 1-ACT PNs under a fluorescent microscope. Only successfully stained animals were included in the analysis.

\section{Results}

Odors elicit activity in PNs in the same glomeruli across animals

Insertion of FURA-dextran crystals into the 1-ACT nerve tract led to a selective staining of PNs (Fig. 2a), as previously reported (Sachse and Galizia 2002). We identified a set of 15 glomeruli in each of the 126 animals included in this study using the digital threedimensional atlas of the honeybee antennal lobe (Galizia et al. 1999a) (Fig. 2a, c).

When stimulating with 1-hexanol, 1-octanol or 1nonanol, PNs showed the same glomerular spatiotemporal activity patterns published previously (Fig. 2b, d) (Sachse et al. 1999; Sachse and Galizia 2002). Hexanol evoked a strong calcium response in glomerulus 28, and weaker responses in glomerulus 17 , 36,38 and 35 , whereas calcium concentration in glomerulus 29 decreased (Fig. 2e). Octanol elicited strong responses in glomeruli 17 and 28 , weak activity in glomerulus 33 and a $\mathrm{Ca}^{2+}$ decrease in glomeruli 23,25 and 29. Nonanol evoked a strong response in glomeruli 17 and 33 and $\mathrm{Ca}^{2+}$ decrease in glomeruli 23 and 25 . All responses were consistent across animals, as can be seen by the small error bars (Fig. 2e). The responses to octanol were overlapping with those to nonanol, while the response to hexanol showed virtually no overlap of activity with nonanol in the 15 observed glomeruli.

No responses could be measured in the AL contralateral to the staining side when stimulating with odors on both antennae (data not shown). This indicates that no bilateral neurons contributed to our signal, notably also not the VUMmx1 neuron. Most likely, these neurons were not labeled with the calcium sensitive dye.

$\mathrm{PN}$ responses to sucrose are similar

between animals

In addition to odors, PNs also responded to sugar water stimulus at the antenna. In Fig. 3 two typical examples of an odor stimulation paired with a sucrose US are shown, one forward (Fig. 3a) and one backward (Fig. 3b). Sucrose solution evoked an activity pattern which was consistent across animals, and identical to stimulation with water alone. The response pattern consisted of several activated glomeruli, suggesting a combinatorial representation as known for odors. The response pattern to water and sucrose solution included glomeruli that also respond to volatile odors (such as glomerulus 17) and glomeruli for which no volatile odor is yet known as a suitable stimulus (such as glomerulus 42 ). The strongest activity was in glomerulus 42 and the lowest was in 33 (Fig. 3c). PNs responded only to sucrose solution when stimulated on the ipsilateral antennae, but not when stimulated at the contralateral antenna or at the proboscis (data not shown). Sucrose responses were clearly distinguishable from mechanical stimulation (data not shown).

Repeated odor stimulation does not change its representation

All 54 animals of set 1 (see Fig. 1) received two "pre" nonanol presentations. PN odor responses in glomerulus 17 and 33 across animals were indistinguishable for the two "pre" stimuli (Fig. 4a). The same held true for all other glomeruli (RM two-way ANOVA, HolmsSidak multiple comparisons, smallest $P=0.059$ for glomerulus 17 , all other $P>0.1$ ).

It is necessary to know the response variability to repeated odor stimulation alone before investigating learning effects. Six successive nonanol stimulations $\left(1 \mathrm{~N}^{-}\right.$group, Fig. $4 \mathrm{~b}$ ) over the $21 \mathrm{~min}$ of the experiment elicited responses that remained unchanged in both magnitude (ANOVA) and shape (visual inspection) in all glomeruli (see responses to glomeruli 17 and 33 in Fig. 4c).

Absolute conditioning does not change the representation of the learned odor

The glomerular odor responses were not statistically different at any point in time (time) between the dif- 
Fig. 2 Odor responses in the honeybee AL. a Raw

fluorescence image of an AL stained with FURA. b Falsecolor coded spatial response patterns to 1-hexanol, 1octanol and 1-nonanol. Glomerulus 17, 28 and 33 are circled with a dotted white line. $\mathbf{c}$ Schematic view of the AL with the 15 identified glomeruli used in this study. d Mean time traces of odor responses in selected

glomeruli ( $n=72$ animals).

Time trace colors correspond to glomeruli in c. Stimulus is marked by the shaded area.

e Odor responses to

1-nonanol, 1-octanol and 1-hexanol for 15 glomeruli ( \pm SEM, $n=72$, average during the $3 \mathrm{~s}$ odor stimulation). The three most responsive glomeruli for these odors are indicated by colored squares

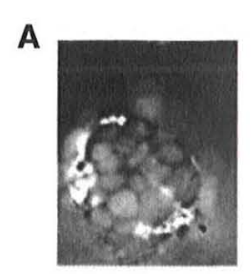

B

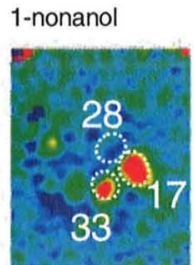

C



D

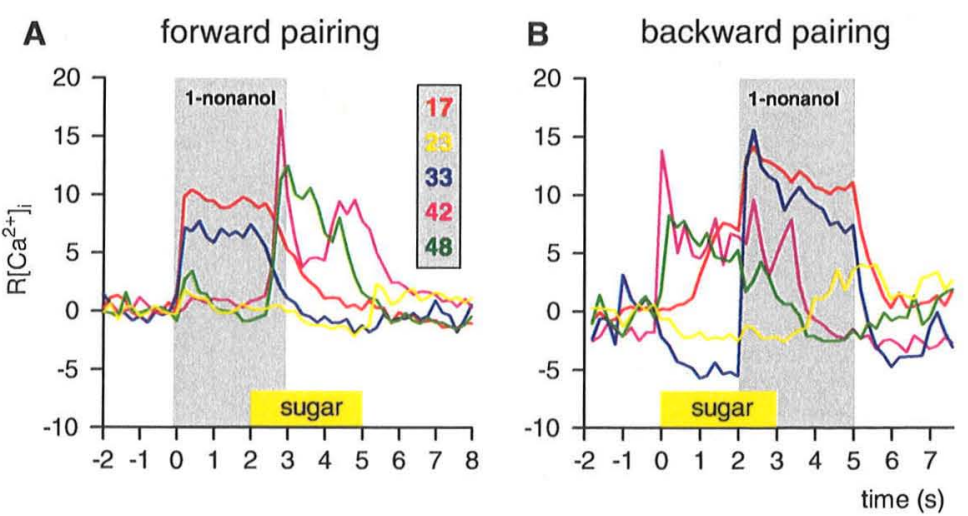

C maximum response to sucrose

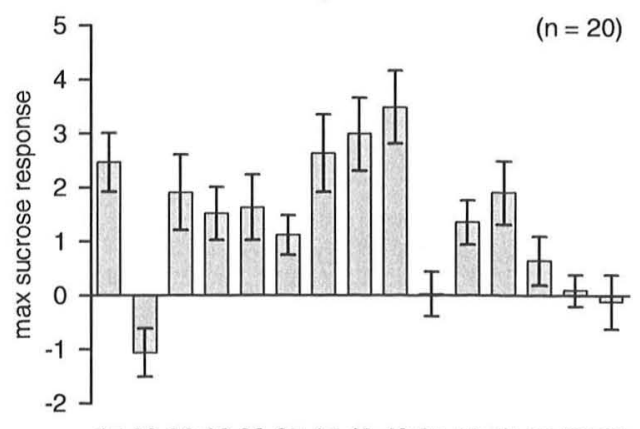

173328363835474842256049372329 glomeruli

Fig. 3 PN responses to sucrose and nonanol. Time traces in five glomeruli of an individual measurement for forward pairing (a) and backward pairing (b). The odor-stimulus (nonanol) is marked by a shaded area, sucrose is marked by the yellow bar. c Averages of maximum responses for 15 identified glomeruli during sucrose stimulation $( \pm$ SEM, $n=20)$ 
Fig. 4 Unrewarded odor responses are stable over time. a Calcium responses in two identified glomeruli, 17 and 33 , to the first and second 1-nonanol stimulation (mean time trace of all 54 animals of set 1 ). The odor-stimulus is marked by a shaded area. ITI $=2$ min. Inset shows average odor responses ( \pm SEM, $n=54$ ) during stimulation. b Group $1^{-}$, average responses $( \pm$ SEM, $n=9$ ) of 15 identified glomeruli to six consecutive 1nonanol stimulations. c

Average time traces (group $1^{-}$) for glomeruli 17 and 33
A glomerulus $17(n=56)$

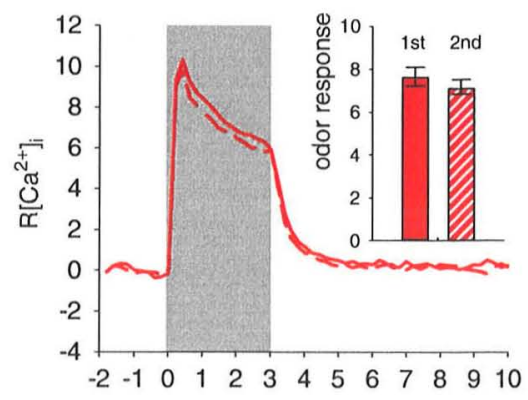

glomerulus $33(n=56)$

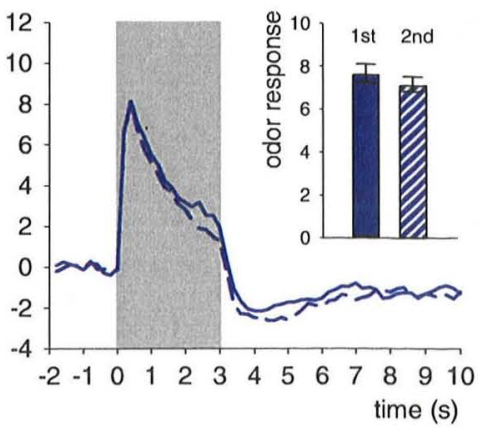

B $\operatorname{group} 1^{-}(\mathrm{n}=9)$

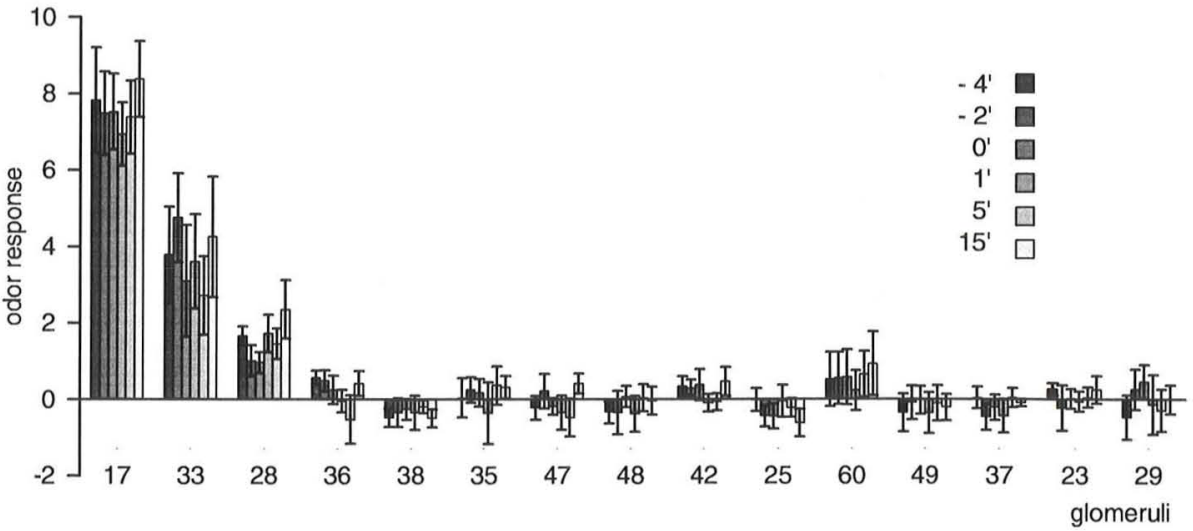

C glomerulus 17 , group $1^{-1}(n=9)$

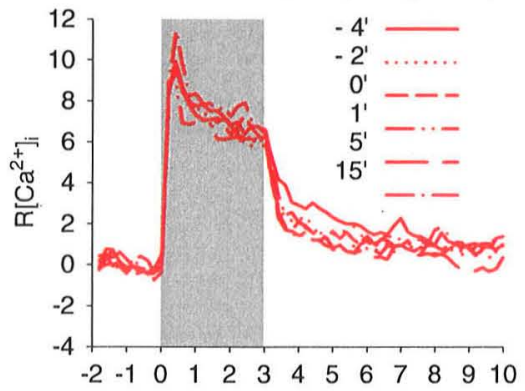

glomerulus 33, group $1^{-}(n=9)$

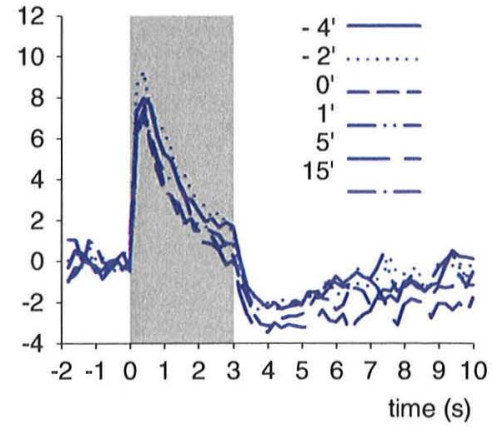

ferent stimulus protocols (group) in set 1 (Fig. 1), i.e. successive odor stimulation, single and multiple trial conditioning, backward pairing and forward pairing (Fig. 5) (no significant interaction between time and group, RM two-way ANOVA, lowest $P$ for glomerulus 17: $d f=16, F=1.614, P=0.067$, in all other cases $P>0.3)$. Therefore, absolute appetitive conditioning did not change the neural representation of the learned odor in 1-ACT projection neurons.

Even in the absence of learning-related modulations, odor responses might be modulated in a learn- ing-independent way, i.e. without a statistical significance for learning paradigm, but common across all groups. Such changes are correlated with experimental time, and not with treatment. Indeed, pooling all animals, we found significant differences in some odor responses. Responses to nonanol at $1 \mathrm{~min}$ after training were attenuated in glomerulus 17 (Holms-Sidak, $P=0.001)$ and the inhibition in glomerulus 38 was reduced $(P=0.024)$; at $15 \mathrm{~min}$, odor responses in glomerulus 38 showed less inhibition $(P=0.001)$, whereas glomeruli $36(P=0.015)$ and $42(P=0.03)$ were 
Fig. 5 Neural representation remains stable following sucrose reinforcement. PN response traces of glomerulus 17 and 33 to 1 -nonanol before conditioning ("pre") compared with responses ("post") at 1, 5 and $15 \mathrm{~min}$ following conditioning. The third column shows the average odor responses of glomerulus 17 and 33 ( \pm SEM, $n$ is given in each graph)
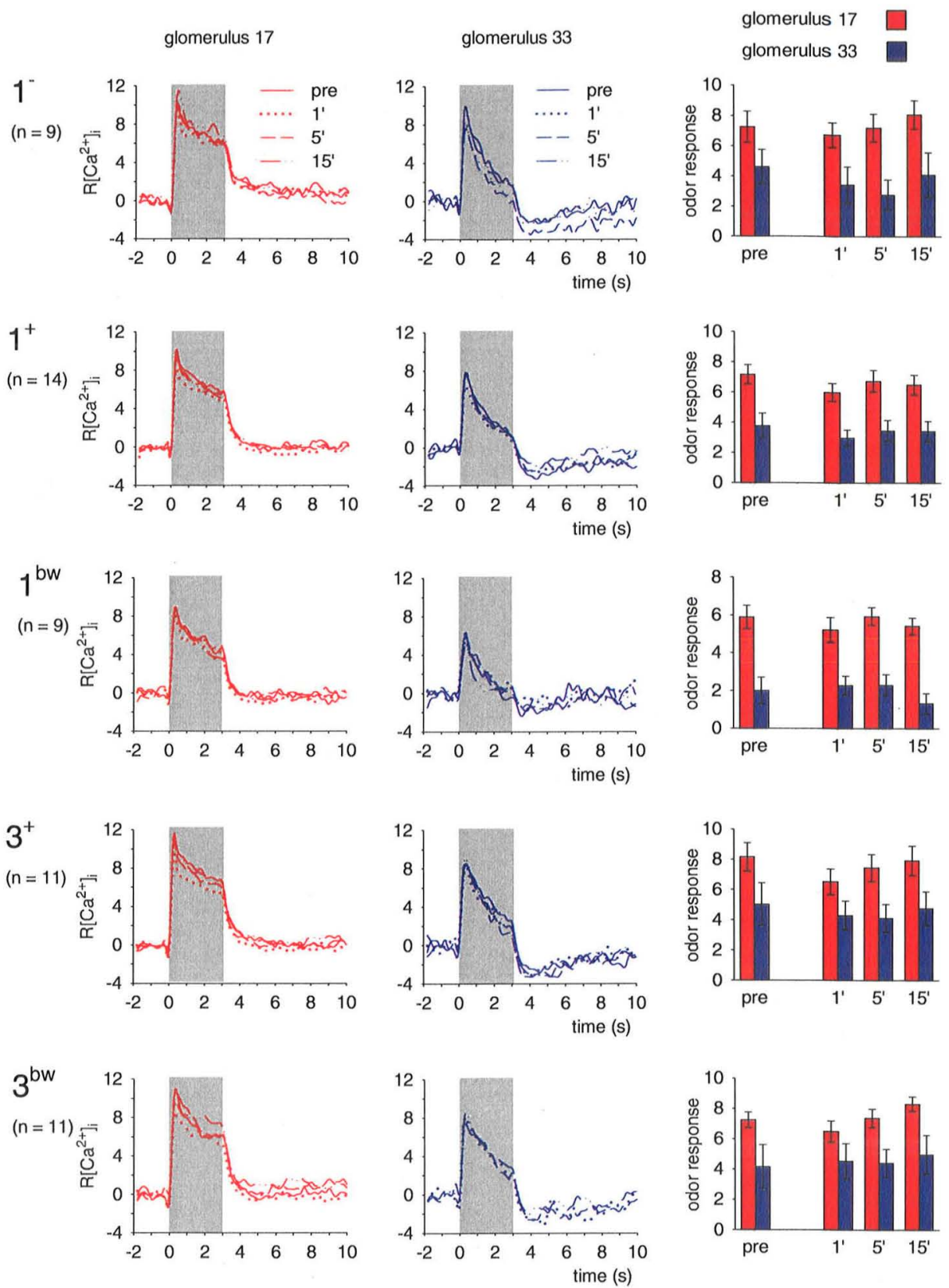

slightly increased (data not shown). All other glomeruli remained unchanged compared to the "pre" at all "post" time points (RM two-way ANOVA).

Both forward and backward pairing leave the learned odor unchanged

While single trial conditioning leads to short-term memory only, 3-trial forward pairing leads to long-term learning and to a prolonged elevation of PKA activity in the AL which is necessary for this long-term mem- ory induction (Müller 2000b). Three-trial backward conditioning, however, does not lead to learning nor to a PKA increase. Therefore, we investigated the responses to the CS-US pairing during multiple trial conditioning. When comparing forward (group $3^{+}$) with backward conditioning (group $3^{\text {bw }}$; Fig. 6), it was not possible to compare responses to the CS only, because CS and US overlap in different ways. For example, during the first second of the CS stimulation the backward group already includes the US (group $3^{\text {bw }}$ ), while the forward group includes the US only 
during the last second (group $3^{+}$) (Fig. 3). We therefore analyzed the combined response to CS and US during the conditioning trial (see Methods). Compound responses to the CS and US did not differ between the two paradigms (smallest $P$ for glomerulus 48 with $d f=1, F=1.972, P=0.176$ two-way RM ANOVA). There was no difference between successive trials (smallest $P$ for glomerulus 25 with $d f=2, F=1.399$, $P=0.259$ ) and no significant interaction between the paradigms and trials (smallest $P$ for glomerulus 29 with $d f=2, \quad F=1.441, \quad P=0.295$ ), with one exception: Glomerulus 60 showed a difference in trial $(d f=2$, $F=3.355, P=0.045$ ) and a Holms-Sidak multiple comparison revealed that trial 2 was different from both, trial 1 and trial 3 ( $P=0.03$, traces not shown). There was no significant interaction between trial and paradigm $(P=0.301)$ and in both groups the second trial was slightly reduced. Glomerulus 60 neither showed a pronounced response to nonanol nor to sucrose stimulation. We therefore regard this significant value as a statistical effect typical for multiple comparison studies and as biologically not relevant.

\section{A backward, group $3^{\text {bw }}(n=11)$}

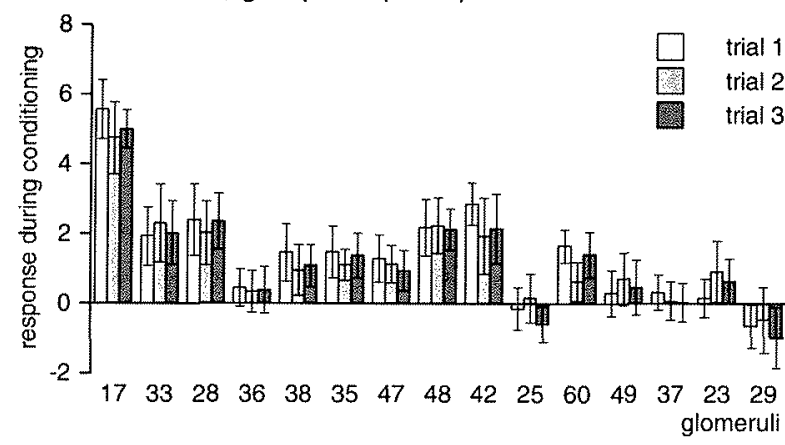

B forward, group $3+(n=11)$

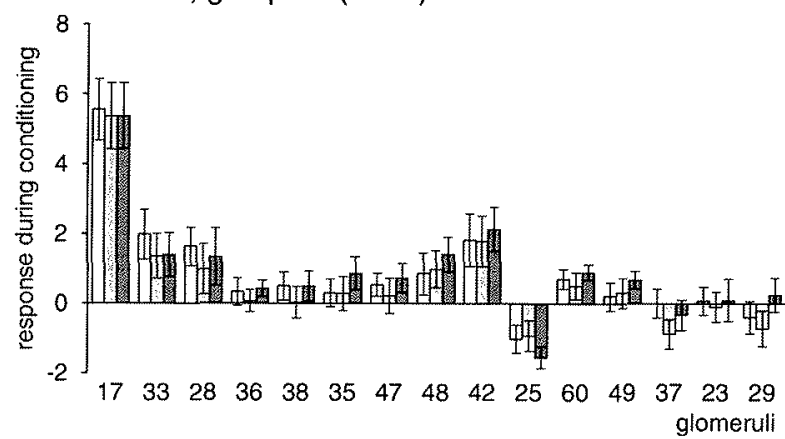

Fig. 6 Responses are stable during conditioning. The compound response to $I$-nonanol and sucrose is not modulated during multiple trial conditioning. a Average compound responses ( \pm SEM, $n=11$ ) during trial 1,2 and 3 of backward conditioning (group $3^{\text {bw }}$ ). b The same for forward conditioning (group $3+$, $n=11$ )
Appetitive conditioning does not change responses to multiple other odors (set 2)

Having established that olfactory conditioning does not change the representation of the conditioned odor, we wondered whether other odors might be modified by learning. To this end, we tested odor responses to three odors before and after training, and conditioned nonanol alone or differentially against octanol or hexanol (set 2, Fig. 1). Statistical analysis of the three "pre" odor responses and the three "post" odor responses revealed no significant differences within "pre" or within "post" (ANOVA for all odor responses in each glomerulus, $d f=2$, smallest $P=0.079$ ). For statistical analysis, therefore, the average of all three responses preceding the treatment was defined as the "pre" odor response for every odor and likewise for the "post" odor response.

There was no significant effect of the conditioning paradigms (group) on any of the odor responses (time) (i.e. the CS+, the CS- or the neutral odor; RM two-way ANOVA, smallest $P$ with $d f=6, F=2.152, P=0.059$, all other $P>0.1$ ). There was no significant interaction between group and time. In more detail, the different paradigms gave the following results. Odor response for the CS+ and the neutral odors were unchanged after five trials of absolute conditioning (group $5 \mathrm{~N}^{+}$; Fig. 7, compare with $3^{+}$, Fig. 5), as shown for the responsive glomeruli 17,28 and 33 in Fig. 7.

Differential conditioning of nonanol against another odor also did not induce any changes in the responses of any of the odors involved. Responses to $\mathrm{CS}+, \mathrm{CS}-$ or the neutral odor (groups $5 \mathrm{~N}^{+} \mathrm{O}^{-}$and $5 \mathrm{~N}^{+} \mathrm{H}^{-}$, Fig. 8) did not change, even for odors with highly overlapping glomerular patterns (group $5 \mathrm{~N}^{+} \mathrm{O}^{-}$). Responses did also not change in those groups which received the same odor sequence but without rewarding nonanol (groups $5 \mathrm{~N}^{-} \mathrm{O}^{-}$and $5 \mathrm{~N}^{-} \mathrm{H}^{-}$, Fig. 8).

Even if odor responses are unchanged after conditioning as compared to before conditioning, odor responses might change during the repeated learning trials. In this analysis it is important to also include CS+and CS-, because in behavioral studies of differential conditioning the first reinforcement of the CS+ leads to an enhanced response to the subsequent CS-, even though the CS- is not paired with the US, indicating that in this condition animals generalize between CS+ and CS-. As differential training progresses, the response to the CS- returns to the spontaneous response level, or falls below it (Bitterman et al. 1983). We therefore compared the CS+ and $\mathrm{CS}-$ responses during conditioning (where generalization would be expected) with the respective 
A 1-nonanol, group $5 \mathrm{~N}^{+}(\mathrm{n}=15)$
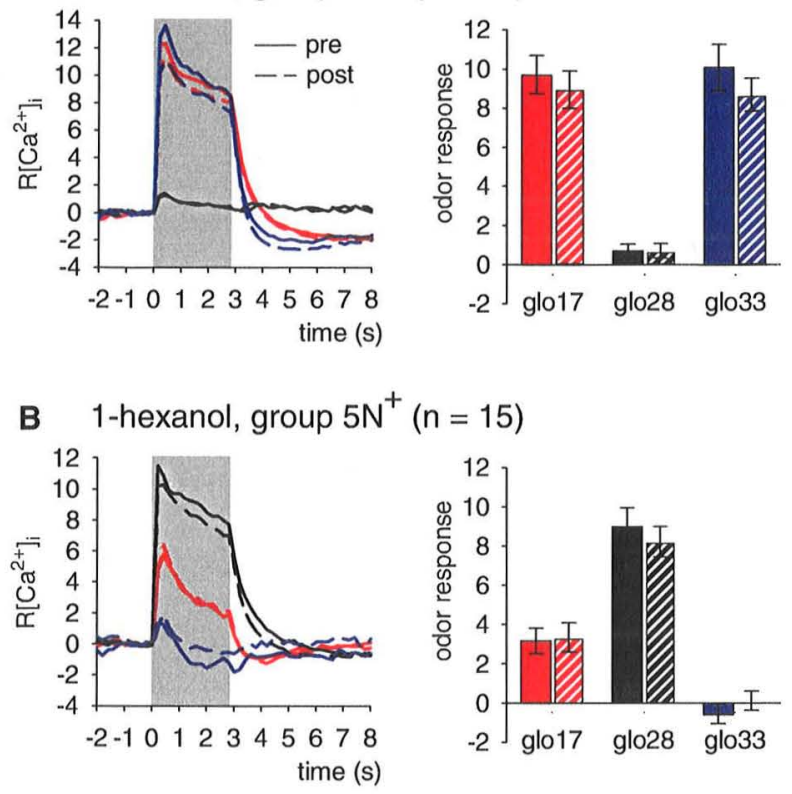

C 1-octanol, group $5 \mathrm{~N}^{+}(n=15)$

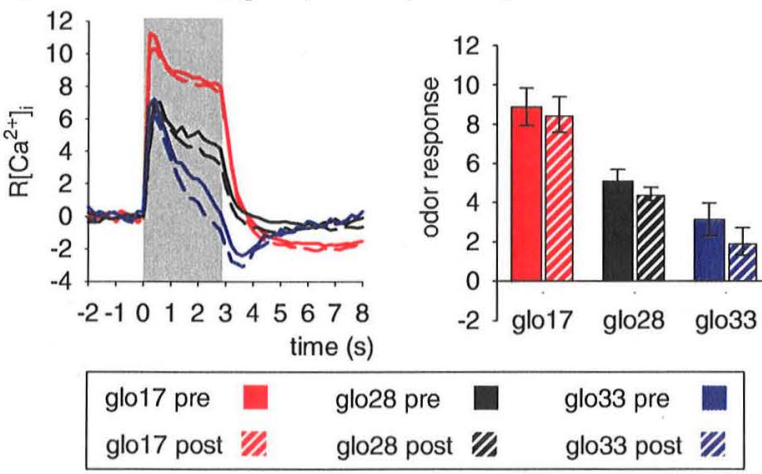

Fig. 7 Absolute conditioning to 1-nonanol does not change the responses to other odors. a Group $5 \mathrm{~N}^{+}$, the average response traces of glomeruli 17, 28 and 33 to 1-nonanol are shown on the left. Solid lines show "pre" response, dotted lines show "post" responses after conditioning. Mean odor responses are plotted on the right ( \pm SEM, $n=15$ ). b The same for responses to 1 hexanol. c The same for responses to 1-octanol

groups that received a similar but unrewarded odor sequence (where no generalization is expected). Because during training odor responses and sugar responses overlap, the analysis had to be modified. For quantification, we took the first second of the odor response to avoid overlap with the sucrose response (group $5 \mathrm{~N}^{+} \mathrm{O}^{-}$and $5 \mathrm{~N}^{-} \mathrm{O}^{-}$, Fig. 9). The different paradigms had no significant effect on any of the odor signals in different trials (RM two-way ANOVA, and no significant interaction between the factors, $d f=4$, all $F<2.168$, all $P>0.083$ ).
Olfactory responses in a multidimensional coding space

Even if individual glomeruli are not significantly modified by learning, slight changes in many glomeruli might add up to a significant across-glomeruli modification relevant for olfactory coding. We therefore analyzed the data using multidimensional statistics. As a simplification, consider a three-dimensional space where each dimension is given by one glomerulus. In such a space, each odor response corresponds to a loop through this space, starting at background, traveling outwards to response peak, and than back to background, close to the coordinate system's origin (Fig. 10a). Odor similarity can then be quantified by the distance between points in this space (Fig. 10b). Mathematically, it is easy to include the data from all available glomeruli, and calculate the Euclidean distance $d_{i j}$ between each odor pair $i j$ as:

$d_{i j}=\sqrt{\sum_{k=1}^{n}\left(x_{i k}-x_{j k}\right)^{2}}$,

where $n$ is the number of measured glomeruli (corresponding to the dimensionality of the Euclidean space, in our case 15), and $x_{j k}$ is the calcium response at $200 \mathrm{~ms}$ to odor $j$ in glomerulus $k$.

Using this statistic, we found that neither differential conditioning, absolute conditioning nor successive odor repetitions had any significant effect on the distances between odor pairs comparing "pre" and "post" odor responses (two-way ANOVA, $d f=6$, $F=1.379, P=0.237)$. The results for groups $5 \mathrm{~N}^{+}$, $5 \mathrm{~N}^{+} \mathrm{O}^{-}$and $5 \mathrm{~N}^{-} \mathrm{O}^{-}$are shown in Fig. 10 .

\section{Comparing effects in individual animals}

All analyses so far addressed the question of whether olfactory learning leads to changes in odor coding that are consistent across animals. However, this leaves open the possibility that the effects might be different in each animal. For example, learning an odor could lead to more glomeruli being activated by that odor, but if these glomeruli were different ones in each individual the effect might not be significant when pooled across animals. Therefore, we analyzed changes in responses to odors for each animal individually.

We defined a glomerulus to give an increased response when and only when each of the three "post" responses were higher than each of the three "pre" responses, and vice-versa for a decrease. We found that several glomeruli had either increased or decreased their responses, as shown in detail for group $5 \mathrm{~N}^{+} \mathrm{O}^{-}$in 
Fig. 8 Differential conditioning does not change odor responses.

a Differentially rewarded group $5 \mathrm{~N}^{+} \mathrm{O}^{-}$, first row: (CS+) 1-nonanol, second row: (CS-) 1-octanol, third row: neutral odor 1-hexanol. Left: mean odor responses ( \pm SEM) before ("pre"-white) and after ("post"- grey) differential conditioning for all glomeruli, right: corresponding average time courses for glomeruli 17, 28 and 33. The odor-stimulus is marked by a shaded area. b The same for group $5 \mathrm{~N}^{-} \mathrm{O}^{-}$, animals were unrewarded but received the same odor sequence as $5 \mathrm{~N}^{+} \mathrm{O}^{-}$in $\mathbf{a}$
A

CS+

1-nonanol



CS-

1-octanol

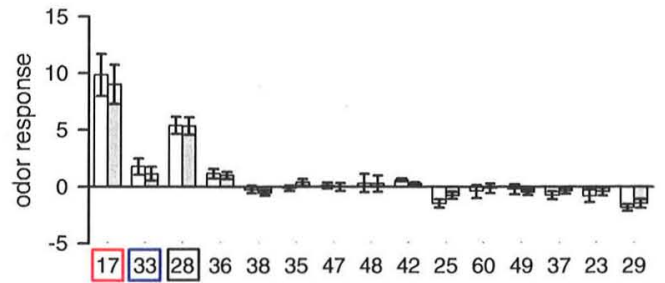

neutral odor

1-hexanol

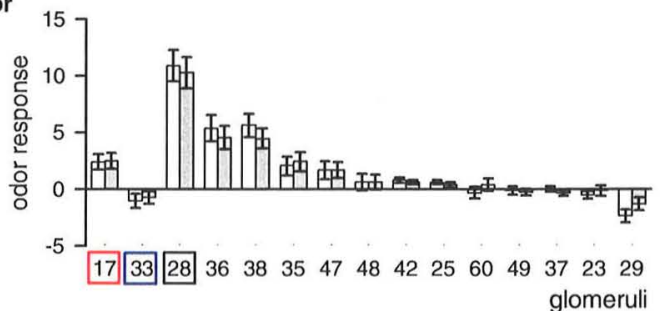

B

1-nonanol

unrewarded, similar odor sequence, group $5 \mathrm{NO}^{-}(\mathrm{n}=10)$
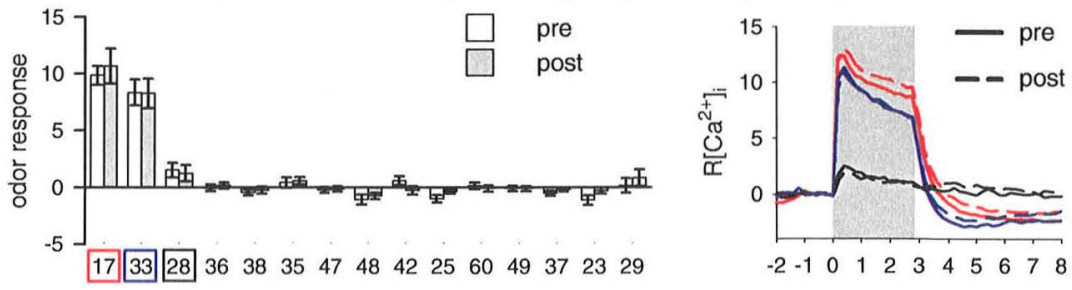

1-octanol
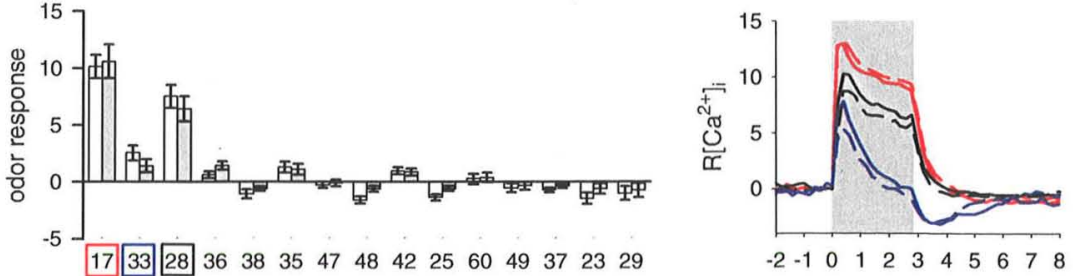

1-hexanol

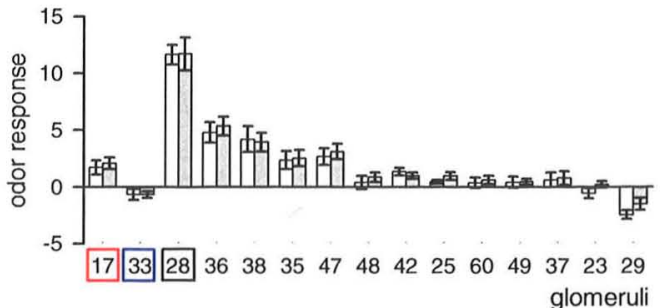

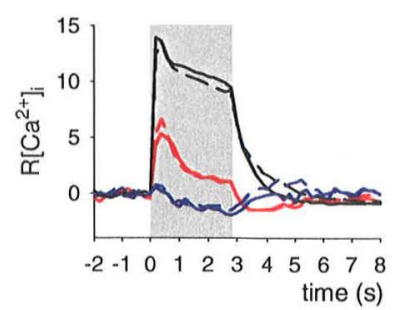


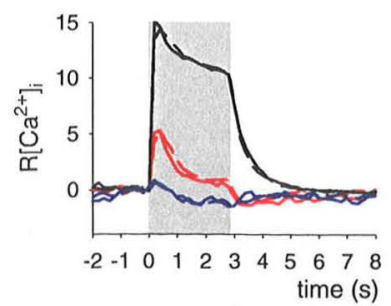

Fig. 11a. However, there was no difference in the number of glomeruli that changed between the CS+ and the CS- (Fig. 11b, showing the differentially trained groups $5 \mathrm{~N}^{+} \mathrm{O}^{-}$and $5 \mathrm{~N}^{+} \mathrm{H}^{-}$, and their respective unrewarded control groups $5 \mathrm{~N}^{-} \mathrm{O}^{-}$and $5 \mathrm{~N}^{-} \mathrm{H}^{-}$). The average number of increased glomeruli for the 
Fig. 9 During differential conditioning odor responses remain unchanged. The five odor responses during five trial differential conditioning to 1-nonanol as the CS+ (upper row) and 1-octanol as the CS-(lower row). On the left are the conditioned animals and on the right the control animals, which received the same odor sequence but no reward. Traces for glomeruli 17, 28 and 33 are averaged across animals, the odor-stimulus is marked by a shaded area. Insets give mean responses during odor stimulation $( \pm$ SEM)

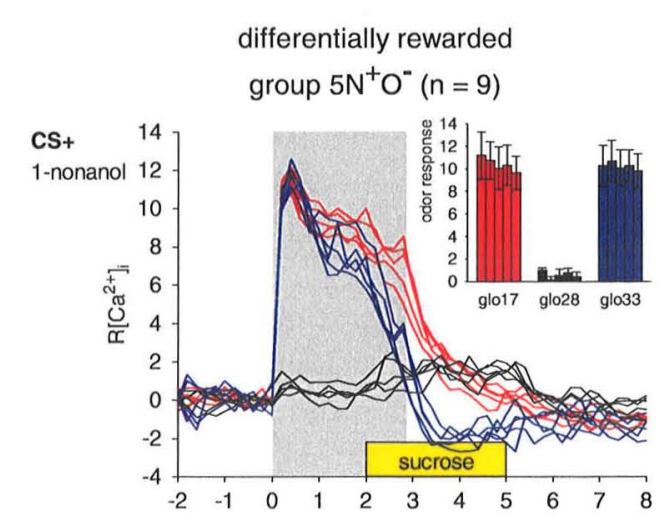

unrewarded, similar odor stimulation group $5 \mathrm{NOO}^{-}(\mathrm{n}=10)$

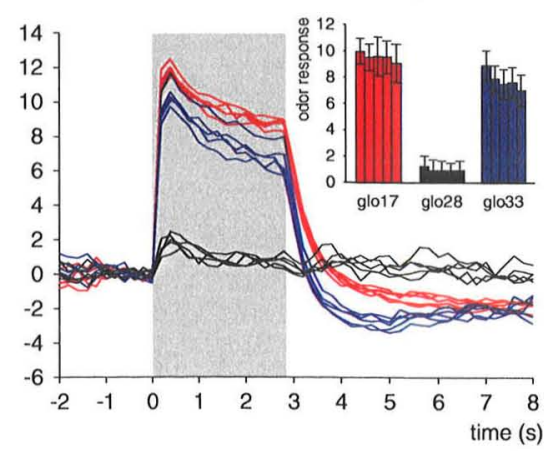

cs-
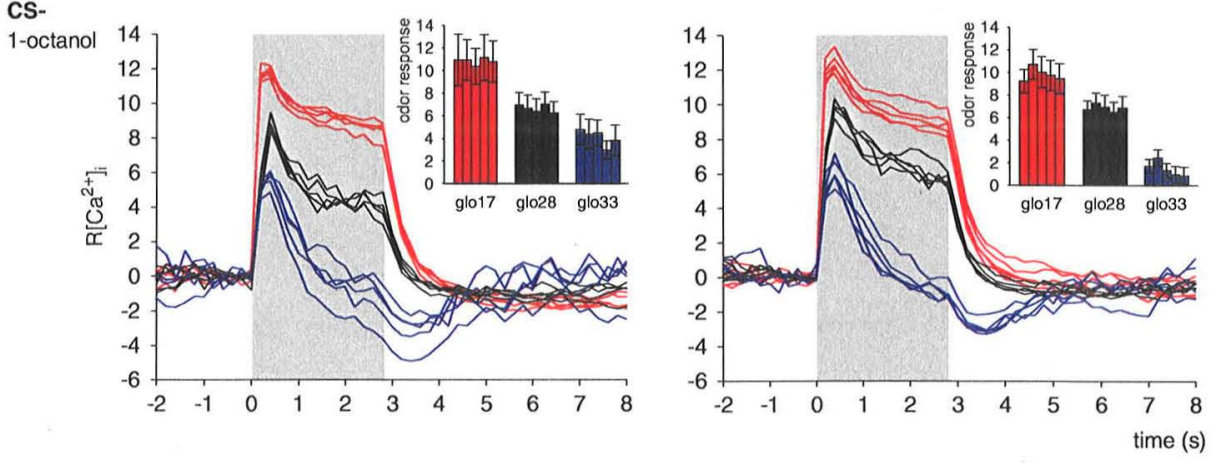

Fig. 10 Odor responses in a multidimensional coding space. Response trajectory to 1-nonanol (red), 1-octanol (blue) and 1-hexanol (black) in a three-dimensional space spanned by glomeruli 17,28 and 33. Dashed lines show responses after $200 \mathrm{~ms}$. b Schematic drawing of the odor response vectors at 200 ms. c Pairwise Euclidian distances between the three odor vectors using all glomeruli, for "pre" responses and "post" responses. Shown are the groups $5 \mathrm{~N}^{+} \mathrm{O}^{-}, 5 \mathrm{~N}^{+}$and $5 \mathrm{~N}^{-} \mathrm{O}^{-}$
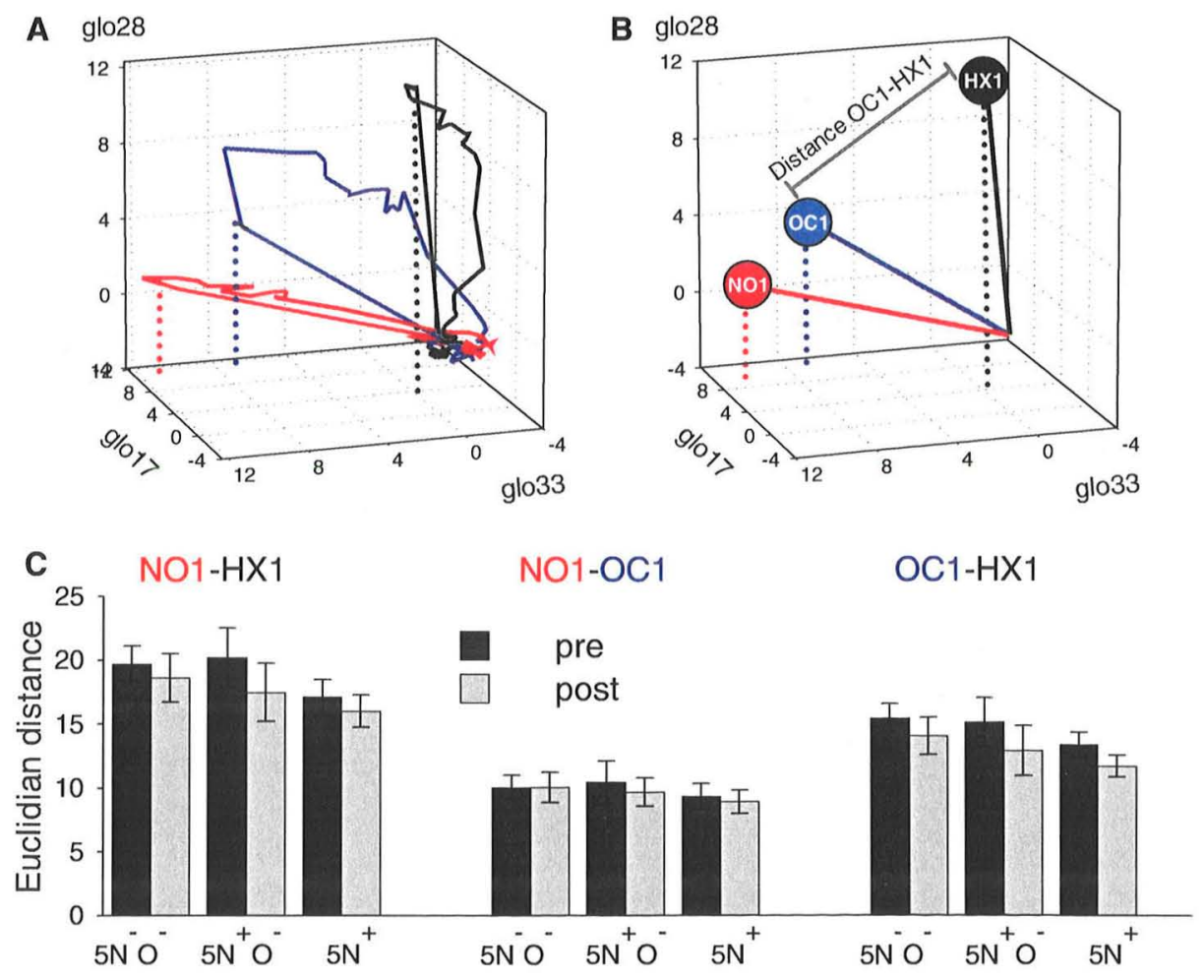
$\mathrm{CS}+, \mathrm{CS}-$ and neutral odor was not different between trained and unrewarded animals.

Plasticity of PN odor responses in the $\mathrm{AL}$

Even if changes in individual odor responses are not related to learning, they still represent a change and therefore might jeopardize reliable odor identification by the animal. We therefore sought to understand the logic of these learning-independent changes. To this end, we pooled all data across conditioning paradigms (Fig. 12). There were a considerable number of glomerular odor responses that changed from "pre" to "post" responses (detailed statistics in Fig. 12), but note that in this analysis all groups were pooled, and these significant effects were not related to learning. Generally, inhibitory responses became less inhibitory (e.g. glomerulus 23 to hexanol: "pre" $=-0.657 \pm 0.08$, "post" $=0.0177 \pm 0.08, d f=1, F=5.122, P<0.001)$ and excitatory responses decreased (e.g. glomerulus 28: "pre" = $10.209 \pm 0.159, \quad$ "post" = 9.671 \pm 0.159 , $d f=1, F=5.727, P=0.020)$. Because these effects were not related to learning, they might be due to a run-down during the experiment (either of the animals, or of the calcium sensitive dye). The (weak) odor response to octanol in glomerulus 36 did not follow this pattern ("pre" $=0.588 \pm 0.0789$, "post" $=1.111 \pm$ $0.0789, d f=1, F=21.984, P<0.001)$. Interestingly, the most prominent glomeruli 17 and 33 for octanol as well as 17 for nonanol did not decrease their response over time.

The role of 1-ACT PNs in PER to odors and sucrose

All physiological measurements of this study were done on PNs of the 1-ACT tract, which are but a subpopulation of all PNs. We therefore investigated the role that these PNs play in odor-coding and odor-driven behavior in a behavioral experiments. For this experiment, animals were prepared and PNs stained just as for an imaging experiment, with the only difference of leaving the muscles responsible for PER intact and the proboscis free to move. Inserting a dye crystal into the 1-ACT disrupts the tract by cutting it. Nevertheless, $95 \%$ of the animals responded to the sucrose with a PER, no matter whether the stimulus was applied to the antenna ipsilateral to the stained PNs, or to the contralateral antenna (Fig. 13a). This shows that the US pathway across the AL had remained intact also on the side where the 1-ACT was damaged by dye injection. After three trial absolute conditioning to nonanol, $65 \%$ of the animals responded with a PER to the CS, showing that the animals learned the olfactory task. However, when the CS was presented unilaterally on the antenna ipsilateral to the stained PNs, the animals did not respond with a PER, indicating that an intact 1-ACT is necessary for odor-recognition, or that olfactory learning was dis-

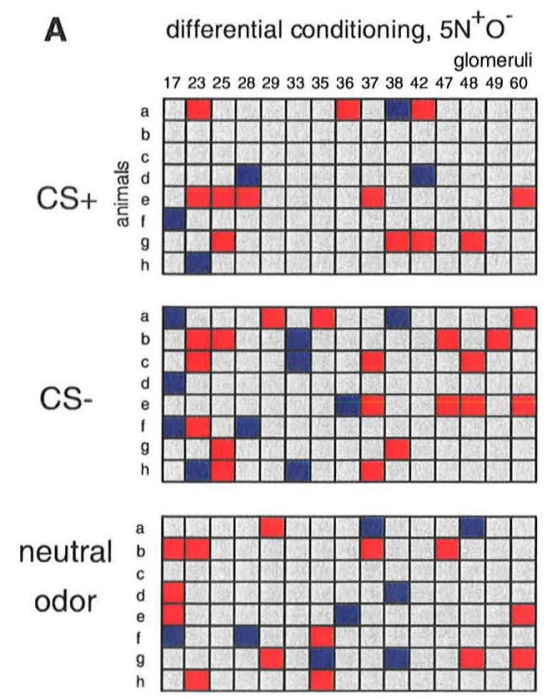

Fig. 11 Occasional response changes are not related to associative learning. a Comparison between "pre" and "post" odor response to the CS+, CS- and the neutral odor in the group $5 \mathrm{~N}^{+} \mathrm{O}^{-}$. Increases are depicted in red (all three "pre" $<$all three "post"), decreases are depicted in blue (all three "pre" > all three "post") for each of 15 glomeruli in eight animals. Gray
B Average number of changing glomeruli



indicates no change. b Summary for differentially conditioned animals (groups $5 \mathrm{~N}^{+} \mathrm{O}^{-}$and $5 \mathrm{~N}^{+} \mathrm{H}^{-}$combined, $n=19$ ) and their control groups (groups $5 \mathrm{~N}^{-} \mathrm{O}^{-}$and $5 \mathrm{~N}^{-} \mathrm{H}^{-}$combined, $n=19$ ): average number of glomeruli showing a change in response to $\mathrm{CS}+, \mathrm{CS}-$ and the neutral odor $( \pm \mathrm{SEM})$. There are no significant changes related to learning 

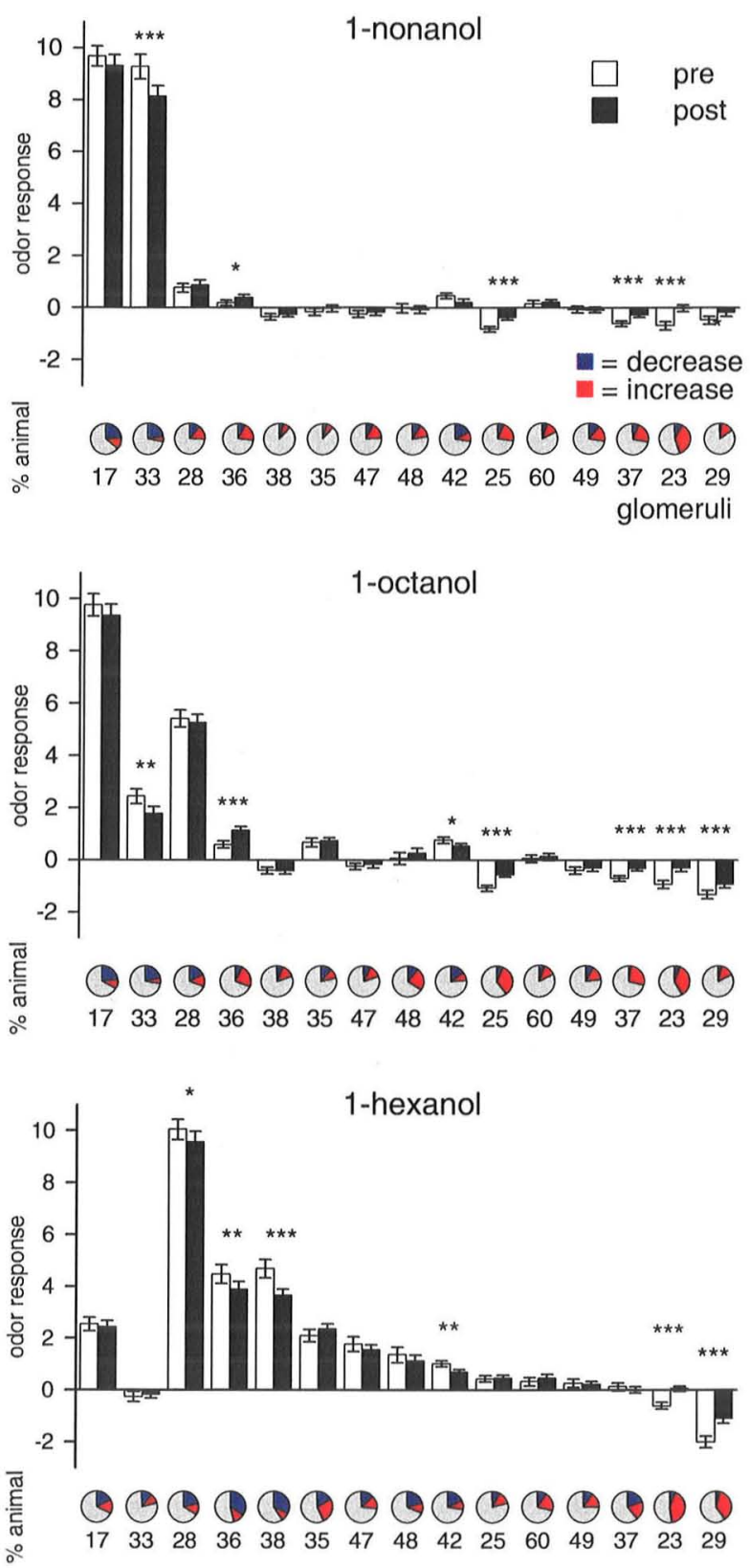

Fig. 12 Plasticity of PN odor responses in the AL. Mean odor responses for all animals to 1-nonanol, 1-octanol and 1-hexanol in each of 15 glomeruli ( \pm SEM, $n=74$ ). "Pre" and "post" odor responses are now pooled and do not correspond to any specific learning group. Statistically different responses are indicated by asterisks (RM two-way ANOVA, Holms-Sidak, ${ }^{*} P<0.05$. $* * P<0.01, * * * P<0.001)$. Below the bar chart, for every glomerulus the percentage of animals showing an increase (red, all three "pre" < all three "post") or decrease (blue, all three "pre" > all three "post") is depicted in a pie chart. Note that these statistically significant changes in odor responses are not related to learning, since all groups were pooled irrespective of their treatment rupted in the ipsilateral AL, or both (Fig. 13b). Unilateral stimulation of the intact side did elicit a PER, showing that the unilateral stimulation itself did not cause the unresponsiveness.

\section{Discussion}

Odors elicit stereotypical activity patterns across olfactory glomeruli in both, vertebrates and invertebrates (Galizia and Menzel 2001; Friedrich 2002). Olfactory learning can modify these patterns (Woo et al. 1987; Sullivan and Wilson 1995; Johnson et al. 1995; Brennan et al. 1998; Faber et al. 1999; Sandoz et al. 2003; Yu et al. 2004; Daly et al. 2004), showing that there is an olfactory memory trace in the vertebrate $\mathrm{OB}$ and in the insect $\mathrm{AL}$. However, this raises an important question. How can olfactory information be processed efficiently in higher order brain centers if the odor code in the primary olfactory centers is subject to change after learning?

Here we report that odor-evoked activity in a subpopulation of honeybee $\mathrm{AL}$ output neurons remains unchanged following appetitive olfactory learning within $15 \mathrm{~min}$ after learning. Since we found that these neurons were necessary for odor recognition in a behavioral experiment, we propose the possibility of parallel olfactory processing in the antennal lobe: 1ACT uniglomerular projection neurons might serve reliable odor-coding, while other PNs may experience memory related plasticity.
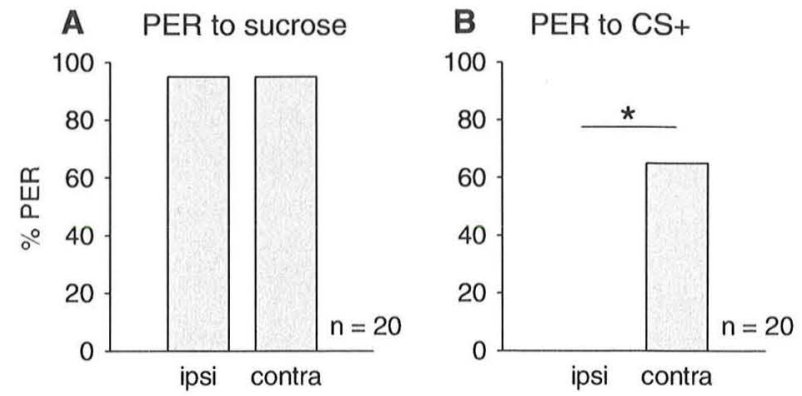

Fig. 13 The 1-ACT-tract is necessary for odor recognition in response to the CS. a Percentage animals which responded to a sucrose stimulation on either the ipsilateral or the contralateral antenna to the stained PNs. b Percentage animals which responded to a learned odor on either the ipsilateral or the contralateral antenna to the stained PNs $\left({ }^{*} P<0.001\right.$, MannWhitney) 
Projection neuron responses to odor and sucrose stimulation

By backfilling PNs from their axonal tracts, we have measured calcium responses in their dendrites within the glomeruli of the AL. This signal reflects the integrated activity in those PNs within the AL and therefore corresponds to activity that has been processed within the AL (Sachse and Galizia 2002). The PN activity that we measure in each glomerulus, however, may be further modified before reaching the PN synapses in the MBs, either by synapses onto PNs at their glomerular exit point (Müller et al. 2002), or presynaptically at the input site to the MB. These modifications are not investigated here. The odor responses that we measured to the odors 1-nonanol, 1-hexanol and 1-octanol corresponded to those previously published for honeybees (Sachse and Galizia 2002), confirming that glomerular odor response profiles are innate to a large extent.

We found that both, water and sucrose stimulation elicited an activity pattern that was equal between animals. We conclude that responses from hygroreceptors (Yokohari et al. 1982; Yokohari 1983) project into the $\mathrm{AL}$ and onto PNs, as shown in cockroaches (Nishino et al. 2003). Honeybees respond with a PER to a sucrose stimulus at the antenna. Since we found no difference between the response to water and sugar, we assume that sugar-receptors do not innervate the glomeruli measured here, in agreement with the finding that sugar-information is relayed to the dorsal lobe (Haupt 2004). However, we cannot exclude that other glomeruli might receive sugar-specific sensory input since we measured but a subset of glomeruli.

Insect brains have several large neurons that branch in many brain areas, and have modulatory function. One of these neurons, the putatively octopaminergic VUMmx1 (Kreissl et al. 1994), is important for olfactory learning and has been shown to mediate the appetitive reinforcement in olfactory PER conditioning (Hammer 1993). It responds to sucrose water stimulation, and branches in the $\mathrm{AL}$, the $\mathrm{MB}$ and the lateral horn. VUMmx1 has a neurite connecting the $\mathrm{AL}$ and the MB that travels close to the $1-\mathrm{ACT}$, and a cell body in the subesophageal ganglion (SEG). We therefore were concerned whether this neuron might have contributed to our signal. However, just as for odors, responses to water and sucrose solution were only observed upon stimulation of the ipsilateral antenna, and not when the contralateral antenna or the proboscis were stimulated. This shows that no signal from modulatory neurons contributed to our measurements. Other, as yet unknown neurons of the bee brain might have been stained by our procedure and could contribute to the signal. Morphological analysis of a few preparations also showed that there was no dye-labeled cell body in the SEG (data not shown). These findings indicate that VUMmx1 has not been damaged by our surgery. Together with the observation that ipsilateral sugar stimulation led to PER response they show that the ipsilateral US-pathways was left intact.

Treated animals learn odors normally, but the 1-ACT is necessary for an ipsilateral response to a $\mathrm{CS}$

In our experiments, animals learned despite being harnessed in a recording chamber, loaded with dye and prepared for imaging (Fig. 13). Thus coincidence detection of US and CS and the motor circuits responsible for PER were intact.

However, when the conditioned odor was applied unilaterally on the side of the stained AL treated animals did not produce a PER. Staining the 1-ACT PNs severed their connection to the MBs. Therefore, this tract is likely to be a necessary part of the odor pathway for odor recognition. This is a very important finding, because the 1-ACT is only one of several PN tracts leading out of the $\mathrm{AL}$, and therefore the data show that there may be no redundancy among tracts: the $\mathrm{m}-\mathrm{ACT}$, which houses uniglomerular PNs from about as many glomeruli as the I-ACT, cannot compensate for the interrupted 1-ACT, although neurons of the two tracts have overlapping response profiles (Müller et al. 2002). In this respect, the situation is different from experiments in rats, where large parts of the $\mathrm{OB}$ can be destroyed without a significant deterioration in olfactory behavior (Lu and Slotnick 1998). The situation also differs from experiments where the MBs were ablated chemically during development, and appetitive conditioning was not affected (Malun et al. 2002).

In all training experiments in this study we applied the odor to both sides, and the sucrose reward to the ipsilateral antenna and to the proboscis. The location of the dye insertion did not disconnect the bilateral reward pathway to the ALs (see above). We therefore conclude that both US and CS information was present in the ALs measured in our imaging experiments, and that learning-induced changes should have occurred to the same extent as in an untreated animal. It is likely that the ipsilateral MB did not learn normally, since its odor-input was blocked by cutting the 1-ACT for dye application, and therefore the CS information was at least partially absent. 
It is conceivable that a centrifugal feedback signal might be necessary in the $\mathrm{AL}$ for learning, in addition to the US and the CS. This centrifugal signal might be missing in our preparation, either because it depends on intact input to the MBs, or because the axons mediating it might have been cut by the dye application, explaining our finding that odor representation did not change. This would imply that three signals have to converge in the AL for learning to take place: the CS, the US and the feedback "instructor" signal. In such a case, the behavioral result that unilateral stimulation with a learned odor did not elicit a response (Fig. 13) could be due to impaired ipsilateral learning, rather than or in addition to a lack in odor recognition. Furthermore, the lack of learning-related changes could also be explained. More research is needed to investigate how such an instructor-signal functions, and if it exists in the first place. Arguing against a necessary role of such a feedback instructor are data that show that bees can also learn to associate an odor with a sucrose reward when the MBs are chemically ablated (Malun et al. 2002). Furthermore, since pairing an odor stimulus with octopamine microinjections into the AL alone is sufficient for associative learning (Hammer and Menzel 1998), we assume that US and CS alone are sufficient for learning. Therefore, learning within the AL was likely not impaired in our study.

\section{Learning does not modify odor representation}

Other studies using different experimental approaches ranging from behavior to molecular biology have shown that the $\mathrm{AL}$ is involved in associative odor learning (Menzel 2001; Davis 2004). Generalization experiments indicate that conditioning to one odor might alter behavioral responses to other odors (Bitterman et al. 1983; Linster et al. 2002; Guerrieri et al. 2005). In rats, odors that elicit almost identical activity patterns in the $O B$ need differential training to be recognized as different chemicals and are generalized after absolute conditioning (Linster et al. 2002). Also in honeybees generalization is reduced after differential olfactory conditioning (Bitterman et al. 1983; Ditzen et al. 2003). This suggests that the boundary between a generalized odor and a discriminated odor can be modified by training, i.e. that the neural olfactory space is plastic. Furthermore, exposure to odors early in development modifies odor responses in the OB (Woo et al. 1987; Johnson et al. 1995). AL morphology changes depending on the task a bee has within the hive community (Winnington et al. 1996). Physiological studies have also shown that physiological properties of AL neurons change after appetitive learning, as shown in honeybees (Faber et al. 1999) and moths (Daly et al. 2004), or after aversive learning, as shown in flies (Yu et al. 2004). All of these experiments suggest that, at the level of the AL, odor coding might be affected by learning.

These changes might be related to the representation of the reinforced odor or to that of the non-reinforced odor, or indeed they might be so subtle that they become only apparent when comparing both at the same time. For this reason, we performed many different training protocols, comprising single and multiple trial learning, with and without additional odors (see Fig. 1). We analyzed the data with respect to odor response magnitude and to odor response time courses for individual glomeruli (Figs. 5, 7, 8). We looked at the global responses simultaneously using a multidimensional analysis based on identified glomeruli (Fig. 10). We also considered statistics of odor response changes while ignoring their glomerular identity (Fig. 11). However, none of these approaches gave any indication of memory-related plasticity. We conclude that olfactory coding is learning-resistant in the population of PNs that we measured, the l-ACT PNs in the AL.

We also found that the sucrose stimulus did not modify the immediate odor response in the AL. Rather, giving an odor and sucrose gave a response corresponding to the superposition of the two individual responses. However, in a pairing condition of a sensory response with a US, we would expect the sensory response to be directly influenced, as is the case in honeybee Kenyon cells in the MBs (Szyszka 2005). This finding therefore argues for a lack of immediate modulatory input to uniglomerular 1-ACT PNs.

\section{Non-associative plasticity of odor responses} in the $\mathrm{AL}$

While PN responses did not change following associative conditioning, we did observe changes that were independent of the conditioning group, and that therefore might indicate a trace of non-associative learning (Fig. 12): during our experiments, the animals experienced the same odors for up to 11 times. We found that responses at the end of the experiments generally had weaker responses in "secondary" glomeruli, i.e. in those glomeruli that were not the strongest glomeruli within the response pattern, and that inhibitory responses were reduced. "Primary" glomeruli (i.e. those with strongest responses) often maintained their response strength. The response to hexanol, where response in glomerulus 28 decreased slightly, might indicate that there could be another 
glomerulus that responds to hexanol with greater affinity than glomerulus 28 , but that was not visible in our experiments.

These changes might optimize the code for the experienced odors, because the contrast between primary and secondary glomeruli increases. However, this change is not related to the "value" of the odor given by associative learning, but entirely to the sensory experience following repeated exposure to the odor. In electrophysiological recordings from locust PNs, repeated stimulation also led to an improved signal-tonoise ratio (Stopfer and Laurent 1999; Bazhenov et al. 2005 ). In a recent study where we also recorded calcium signals from honeybee 1-ACT neurons, we found that odor exposure modifies spontaneous activity patterns in the AL over a few minutes revealing a shortlasting sensory memory (Galán et al. 2006). Whether these findings are due to the same cellular mechanisms remains to be elucidated. These non-associative changes may be related to effects where exposure to an odor modifies subsequent odor-related behavior. For example, repeated odor stimulation leads to a reduced response in subsequent conditioning experiments with the same odor (latent inhibition) (Hammer and Menzel 1995), or unreinforced exposure to an odor mixture prior to conditioning one of its components leads to an associative response also to its other component (preconditioning) (Müller et al. 2000).

Are other neurons subject to modulation in the AL?

Given the lack of plasticity in 1-ACT-PNs in our experiments, we see three possible explanations. First, it is possible that 1-ACT-PNs change temporal properties of their firing patterns without a change in firing rate that would be visible via intracellular calcium changes. Such changes are beyond our measurement technique. Second, the AL might require a feedback signal from the MBs in order to learn, in addition to the CS and the US. We cannot exclude this possibility, though experimental evidences such as pairing an olfactory stimulus with octopamine injections into the AL (Hammer and Menzel 1998) suggest that CS and US might not need an additional signal for learning (see above). A related possibility is that the ipsilateral VUMmx1 collateral to the MB was damaged, and that this damage might interfere with the associative processes at the level of the AL. However, our data argue against this possibility (see above). A third alternative is that indeed 1-ACT-PNs are non-plastic, but other PNs are. It is therefore important, now, to extend our studies to the remaining populations of
PNs. In honeybees, there are three major tracts: the 1ACT investigated here, the m-ACT, and the ml-ACT. $\mathrm{m}$-ACT-PNs are uniglomerular PNs from about 70 glomeruli innervated by the antennal T3 sensory axons with no or little overlap with the 70 glomeruli of the 1-ACT, which are innervated by the antennal $\mathrm{T} 1$ sensory axons. Response properties of $\mathrm{m}$-ACT and 1ACT PNs differ (Abel et al. 2001; Müller et al. 2002). It should be possible to record from $\mathrm{m}$-ACT uniglomerular neurons in a similar way as we recorded from 1-ACT PNs, and to test the hypothesis that all uniglomerular PNs are resistant to changes following appetitive learning.

If that hypothesis is true, and given that there is a memory trace in the AL (Menzel and Muller 1996; Menzel 2001), the neurons to carry that change to other brain areas could be multiglomerular PNs or the uniglomerular PNs in the m-ACT. Multiglomerular PNs have been described for several insects species, e.g., cockroaches (Ernst et al. 1977), Manduca sexta (Kanzaki et al. 1989), Lepidoptera (Anton and Hansson 1994), and Drosophila (Stocker et al. 1990). In honeybees, these neurons do not innervate the calyces of the $\mathrm{MBs}$, but project to the lateral protocerebrum and adjacent areas (Abel et al. 2001). Since these neurons branch in large areas of the $\mathrm{AL}$, they have simultaneous access to the combinatorial odor activity pattern and possibly the US, which could make them candidates for associative odor memory. In the few recordings from honeybee ml-ACT neurons reported so far, multiglomerular PNs have been shown to remain silent to many odors despite innervating a large number of glomeruli (Müller 2000a), or to drastically change their responses during an experiment (Abel 1997). This response behavior suggests that multiglomerular PNs may not code odor-identity, but other information, which might be related to its associative value.

Measuring m-ACT PNs and ml-ACT PNs might solve the apparently conflicting results between our study and others. Changes in the AL in the honeybee were shown using calcium imaging with bath applied dyes (Faber et al. 1999; Sandoz et al. 2003). In those studies, the signal was dominated by receptor neuron input, but contained also an unknown component of other cells, possibly including multiglomerular PNs (Galizia and Vetter 2004). Changes in PN odor responses were shown in Drosophila (Yu et al. 2004). In that study, a genetic line (GH146) was used that labels a combination from several tracts, and includes multiglomerular neurons (Stocker et al. 1997; Marin et al. 2002). Most neurons in GH146 travel in the iACT (Wong et al. 2002), which is located medially and might 
correspond to the honeybee m-ACT tract. This raises the additional possibility that in honeybees uniglomerular PNs in the $\mathrm{m}-\mathrm{ACT}$ might change their odorresponses after learning. Their response profiles overlap and their response dynamics differ from those of the 1-ACT neurons (Müller et al. 2002). Unfortunately, no GAL4 line is known for Drosophila that only labels uniglomerular PNs which do not use the iACT, so that our experiments cannot, at present, be replicated in that model animal. Recordings from moths showed associative plasticity in multi-unit recordings, but again the identity of the recorded neurons was unclear, and may have been any combination of LNs and/or PNs from different tracts (Daly et al. 2004).

Even if both uniglomerular PN tracts in the bee may not be involved in transmitting learning related plasticity at the AL level they might still contribute to such plasticity at the MB calyx level. Higher order brain centers, such as the insect MBs, are strongly involved in olfactory learning (Tully et al. 1994; Menzel 1999; Heisenberg 2003). A system where at least some PNs remain unaffected by learning would appear an efficient solution, because these neurons could provide a substrate for reliable odor-identity information towards the $\mathrm{MBs}$, which need such information for forming their own reliable memories. Other neurons, e.g. multiglomerular PNs, could be plastic, and encode memories at the level of the AL. Whether AL and MB memories differ in timing [short-term vs. long-term as suggested by indirect evidence (Menzel 1990, 1999)] or in quality [e.g. elemental vs. configural and context dependent memories (Giurfa 2003)] remains to be elucidated.

Acknowledgments Support from Volkswagenstiftung, Human Frontier Science Program, Deutscher Akademischer Austauschdienst and Deutsche Forschungsgemeinschaft SFB 515 is acknowledged. Thanks also to Uli Müller for intellectual exchange during the entire project, to Paul Szyszka for valuable discussion and to Silke Sachse, Daniela Pelz and the referees for comments on the manuscript.

\section{References}

Abel R (1997) Das olfaktorische System der Honigbiene: elektrophysiologische und morphologische Charakterisierung von Antennallobus Neuronen und deren beteiligung beim olfaktorischen Lernen. PhD Thesis, FU Berlin

Abel R, Rybak J, Menzel R (2001) Structure and response patterns of olfactory interneurons in the honeybee, Apis mellifera. J Comp Neurol 437:363-383

Anton S, Hansson BS (1994) Central processing of sex pheromone, host odour, and oviposition deterrent information by interneurons in the antennal lobe of female Spodoptera littoralis (Lepidoptera: Noctuidae). J Comp Neurol 350:199214
Bazhenov M, Stopfer M, Sejnowski TJ, Laurent G (2005) Fast odor learning improves reliability of odor responses in the locust antennal lobe. Neuron 46:483-492

Bitterman ME, Menzel R, Fietz A, Schafer S (1983) Classical conditioning of proboscis extension in honeybees (Apis mellifera). J Comp Psychol 97:107-119

Brennan PA, Schellinck HM, de la RC, Kendrick KM, Keverne EB (1998) Changes in neurotransmitter release in the main olfactory bulb following an olfactory conditioning procedure in mice. Neuroscience 87:583-590

Daly KC, Christensen TA, Lei $\mathrm{H}$, Smith BH, Hildebrand JG (2004) Learning modulates the ensemble representations for odors in primary olfactory networks. Proc Natl Acad Sci USA 101:10476-10481

Davis RL (2004) Olfactory learning. Neuron 44:31-48

Deisig N, Lachnit H, Giurfa M, Hellstern F (2001) Configural olfactory learning in honeybees: negative and positive patterning discrimination. Learn Mem 8:70-78

Ditzen M, Evers JF, Galizia CG (2003) Odor similarity does not influence the time needed for odor processing. Chem Senses 28:781-789

Ernst KD, Boeckh J, Boeckh V (1977) A neuroanatomical study on the organization of the central antennal pathways in insects. Cell Tissue Res 176:285-306

Faber T, Menzel R (2001) Visualizing mushroom body response to a conditioned odor in honeybees. Naturwissenschaften $88: 472-476$

Faber T, Joerges J, Menzel R (1999) Associative learning modifies neural representations of odors in the insect brain. Nat Neurosci 2:74-78

Flanagan D, Mercer AR (1989) An atlas and 3-D reconstruction of the antennal lobes in the worker honey bee, Apis mellifera L. (Hymenoptera: Apidae). Int J Insect Morphol Embryol 18:145-159

Friedrich RW (2002) Real time odor representations. Trends Neurosci 25:487-489

Galán RF, Weidert M, Menzel R, Herz A, Galizia CG (2006) Sensory memory for odors is encoded in spontaneous correlated activity between olfactory glomeruli. Neural Comput 18:10-25

Galizia CG, Menzel R (2001) The role of glomeruli in the neural representation of odours: results from optical recording studies. J Insect Physiol 47:115-130

Galizia CG, Vetter R (2004) Optical methods for analyzing odorevoked activity in the insect brain. In: Christensen TA (ed) Advances in insect sensory neuroscience. CRC Press, Boca Raton, pp 349-392

Galizia CG, Joerges J, Kuttner A, Faber T, Menzel R (1997) A semi-in-vivo preparation for optical recording of the insect brain. J Neurosci Methods 76:61-69

Galizia CG, McIlwrath SL, Menzel R (1999a) A digital threedimensional atlas of the honeybee antennal lobe based on optical sections acquired by confocal microscopy. Cell Tissue Res 295:383-394

Galizia CG, Sachse S, Rappert A, Menzel R (1999b) The glomerular code for odor representation is species specific in the honeybee Apis mellifera. Nat Neurosci 2:473-478

Gerber B, Menzel R (2000) Contextual modulation of memory consolidation. Learn Mem 7:151-158

Giurfa M (2003). Cognitive neuroethology: dissecting non-elemental learning in a honeybee brain. Curr Opin Neurobiol 13:726-735

Gluck MA, Granger R (1993) Computational models of the neural bases of learning and memory. Annu Rev Neurosci 16:667-706 
Guerrieri F, Schubert M, Sandoz JC, Giurfa M (2005) Perceptual and neural olfactory similarity in honeybees. PLoS Biol 3:e60

Hammer M (1993) An identified neuron mediates the unconditioned stimulus in associative olfactory learning in honeybees. Nature 366:59-63

Hammer M, Menzel R (1995) Learning and memory in the honeybee. J Neurosci 15:1617-1630

Hammer M, Menzel R (1998) Multiple sites of associative odor learning as revealed by local brain microinjections of octopamine in honeybees. Learn Mem 5:146-156

Haupt SS (2004) Antennal sucrose perception in the honey bee (Apis mellifera L.): behaviour and electrophysiology. J Comp Physiol A 190:735-745

Heisenberg M (2003) Mushroom body memoir: from maps to models. Nat Rev Neurosci 4:266-275

Hildebrand JG, Shepherd GM (1997) Mechanisms of olfactory discrimination: converging evidence for common principles across phyla. Annu Rev Neurosci 20:595-631

Johnson BA, Woo CC, Duong H, Nguyen V, Leon M (1995) A learned odor evokes an enhanced Fos-like glomerular response in the olfactory bulb of young rats. Brain Res 699:192-200

Kanzaki R, Arbas EA, Strausfeld NJ, Hildebrand JG (1989) Physiology and morphology of projection neurons in the antennal lobe of the male moth Manduca sexta. J Comp Physiol A 165:427-453

Kreissl S, Eichmuller S, Bicker G, Rapus J, Eckert M (1994) Octopamine-like immunoreactivity in the brain and subesophageal ganglion of the honeybee. $J$ Comp Neurol 348:583-595

Linster C, Johnson BA, Morse A, Yue E, Leon M (2002) Spontaneous versus reinforced olfactory discriminations. J Neurosci 22:6842-6845

Lohmann C, Wong RO (2005) Regulation of dendritic growth and plasticity by local and global calcium dynamics. Cell Calcium 37:403-409

Lu XC, Slotnick BM (1998) Olfaction in rats with extensive lesions of the olfactory bulbs: implications for odor coding. Neuroscience 84:849-866

Malun D (1991) Synaptic relationships between GABA-immunoreactive neurons and an identified uniglomerular projection neuron in the antennal lobe of Periplaneta americana: a double-labeling electron microscopic study. Histochemistry 96:197-207

Malun D, Giurfa M, Galizia CG, Plath N, Brandt R, Gerber B, Eisermann B (2002) Hydroxyurea-induced partial mushroom body ablation does not affect acquisition and retention of olfactory differential conditioning in honeybees. J Neurobiol 53:343-360

Marin EC, Jefferis GS, Komiyama T, Zhu H, Luo L (2002) Representation of the glomerular olfactory map in the Drosophila brain. Cell 109:243-255

Mauelshagen J (1993) Neural correlates of olfactory learning paradigms in an identified neuron in the honeybee brain. $\mathbf{J}$ Neurophysiol 69:609-625

Menzel R (1990) Learning, memory, and "cognition" in honey bees. In: Kesner RP, Olton DS (eds) Neurobiology of comparative cognition. Erlbaum Inc, Hillsdale, pp 237-292

Menzel R (1999) Memory dynamics in the honeybee. J Comp Physiol A 185:323-340

Menzel R (2001) Searching for the memory trace in a mini-brain, the honeybee. Learn Mem 8:53-62

Menzel R, Giurfa M (2001) Cognitive architecture of a minibrain: the honeybee. Trends Cogn Sci 5:62-71
Menzel R, Muller U (1996) Learning and memory in honeybees: from behavior to neural substrates. Annu Rev Neurosci 19:379-404

Menzel R, Hammer M, Braun G, Mauelshagen J (1991) Neurobiology of learning and memory in honeybees. In: Goodmann LJ, Fisher RC (eds) The behaviour and physiology of bees. CAB International, Wallingford, pp 323-353

Mobbs PG (1982) The brain of the honeybee Apis mellifera I. The connections and spatial organization of the mushroom bodies. Philos Trans R Soc Lond B 298:309-354

Mori K, Nagao H, Yoshihara Y (1999) The olfactory bulb coding and processing of odor molecule information. Science 286:711-715

Müller D (2000a) Die olfaktorische Kodierung: Plastizität und Stabilität-Eine elektrophysiologische Analyse der Ausg. angsneurone des Antennallobus der Honigbiene. PhD Thesis, FU Berlin

Müller U (2000b) Prolonged activation of cAMP-dependent protein kinase during conditioning induces long-term memory in honeybees. Neuron 27:159-168

Müller D, Gerber B, Hellstern F, Hammer M, Menzel R (2000) Sensory preconditioning in honeybees. J Exp Biol 203:13511364

Müller D, Abel R, Brandt R, Zockler M, Menzel R (2002) Differential parallel processing of olfactory information in the honeybee, Apis mellifera L. J Comp Physiol A 188:359-370

Mutoh H, Yuan Q, Knopfel T (2005) Long-term depression at olfactory nerve synapses. J Neurosci 25:4252-4259

Nishino $\mathrm{H}$, Yamashita S, Yamazaki Y, Nishikawa M, Yokohari F, Mizunami M (2003) Projection neurons originating from thermo- and hygrosensory glomeruli in the antennal lobe of the cockroach. J Comp Neurol 455:40-55

Sachse S, Galizia CG (2002) Role of inhibition for temporal and spatial odor representation in olfactory output neurons: a calcium imaging study. J Neurophysiol 87:1106-1117

Sachse S, Rappert A, Galizia CG (1999) The spatial representation of chemical structures in the antennal lobe of honeybees: steps towards the olfactory code. Eur J Neurosci 11:3970-3982

Sandoz JC, Menzel R (2001) Side-specificity of olfactory learning in the honeybee: generalization between odors and sides. Learn Mem 8:286-294

Sandoz JC, Galizia CG, Menzel R (2003) Side-specific olfactory conditioning leads to more specific odor representation between sides but not within sides in the honeybee antennal lobes. Neuroscience 120:1137-1148

Stocker RF, Lienhard MC, Borst A, Fischbach KF (1990) Neuronal architecture of the antennal lobe in Drosophila melanogaster. Cell Tissue Res 262:9-34

Stocker RF, Heimbeck G, Gendre N, de Belle JS (1997) Neuroblast ablation in Drosophila P[GAL4] lines reveals origins of olfactory interneurons. J Neurobiol 32:443-456

Stopfer M, Laurent G (1999) Short-term memory in olfactory network dynamics. Nature 402:664-668

Sullivan RM, Wilson DA (1995) Dissociation of behavioral and neural correlates of early associative learning. Dev Psychobiol 28:213-219

Szyszka P (2005) Odor coding and neural plasticity in the mushroom body of the honeybee. PhD Thesis, FU Berlin

Tanaka NK, Awasaki T, Shimada T, Ito K (2004) Integration of chemosensory pathways in the Drosophila second-order olfactory centers. Curr Biol 14:449-457

Tully T, Preat T, Boynton SC, Del Vecchio M (1994) Genetic dissection of consolidated memory in Drosophila. Cell $79: 35-47$ 
Wilson DA, Stevenson RJ (2003) Olfactory perceptual learning: the critical role of memory in odor discrimination. Neurosci Biobehav Rev 27:307-328

Winnington AP, Napper RM, Mercer AR (1996) Structural plasticity of identified glomeruli in the antennal lobes of the adult worker honey bee. J Comp Neurol 365:479-490

Wong AM, Wang JW, Axel R (2002) Spatial representation of the glomerular map in the Drosophila protocerebrum. Cell 109:229-241

Woo CC, Coopersmith R, Leon M (1987) Localized changes in olfactory bulb morphology associated with early olfactory learning. J Comp Neurol 263:113-125
Yokohari F (1983) The coelocapitular sensillum, an antennal hygro- and thermoreceptive sensillum of the honey bee, Apis mellifera $\mathrm{L}$. Cell Tissue Res 233:355-365

Yokohari F, Tominaga Y, Tateda H (1982) Antennal hygroreceptors of the honey bee, $k$. Cell Tissue Res 226:63-73

Yokoi M, Mori K, Nakanishi S (1995) Refinement of odor molecule tuning by dendrodendritic synaptic inhibition in the olfactory bulb. Proc Natl Acad Sci USA 92:3371-3375

Yu D, Ponomarev A, Davis RL (2004) Altered representation of the spatial code for odors after olfactory classical conditioning; memory trace formation by synaptic recruitment. Neuron 42:437-449 